\title{
Fleming-Harrington Redistribution Wash-in Washout (FHRWW): The Platinum Standard for Nuclear Cardiology
}

\author{
Richard M. Fleming and Gordon M. Harrington \\ FHHI, UNI \\ USA
}

\section{Introduction}

Since its advent in 1925, Nuclear Cardiology has undergone multiple changes in technology including detectors, computers, changes in protocols, various approaches to stress including physiologic and pharmacologic methods, changes in the type of isotope utilized and finally concerns over the distribution of radioactive isotopes and efforts to improve the detection of heart disease while reducing radiation dosage to both patients and health care workers alike. We will divide this chapter into five Sections. First, we will review the work that led to the discovery that the technetium-99m isotopes, like thallium-201, redistribute. Secondly, we will look at currently employed Cardiac imaging using Single Photon Emission Computed Tomography (SPECT) cameras for detecting technetium-99m isotopes (principally Sestamibi). Thirdly, we will consider how these isotopes are injected, distributed and redistributed within Cardiac tissue. Fourth, we will look at how single injection stress-stress imaging can not only increase the accuracy of SPECT imaging of the heart, detecting the most critically diseased coronary vessels while reducing the amount of radioactive isotope injected into the patient, who will subsequently expose others to this radiation and finally, we will look at how rest-rest comparisons of isotope redistribution can be used to differentiate between viable and non-viable damaged myocardial tissue using SPECT cameras.

At the completion of this chapter, clinician and scientist alike, will (1) better understand the physics of SPECT imaging of the heart, (2) will better understand the kinetics of technetium$99 \mathrm{~m}$ isotopes (which are applicable to thallium-201 and other isotopes, given differences in redistribution times), (3) be able to better detect heart disease with special emphasis placed on ischemic heart disease, while addressing issues of patient radiation safety (4) be able to differentiate viable from non-viable cardiac tissue and (5) be able to utilize SPECT cameras to extract both anatomic and physiologic information, not currently possible with single isotope injection, single camera imaging systems, including Positron Emission Tomography (PET), Magnetic Resonance Imaging (MRI) and/or Computed Tomography (CT). Currently, imaging with PET cameras, cannot be completed rapidly enough to allow for measurements of redistribution properties of PET isotopes, nor is it clear if any of these isotopes (ammonia, FDG, Rb-82, etc) actually have redistribution properties, while MRI and Coronary CT currently are only able to yield anatomic information and are unable to measure physiologic redistribution of isotopes. This chapter will describe the recent 
advances in single isotope injection, single camera imaging (FHRWW) while explaining why the practice of rest-stress imaging has failed to deliver on the promises made.

\section{Discovering the redistribution properties of technetium-99m isotopes}

The development of Nuclear Cardiology (discussed in detail later in this chapter) evolved through the utilization of Thallium-201 (Tl-201) by the 1970's. The protocol at that time consisted of stressing the patient and injecting 1-3 mCi (74-111 MBq) intravenously into the patients venous system and continuing to exercise the patient for an additional minute prior to ceasing exercise (Fleming, 1991a, 1999a). The patient was then "recovered" and underwent "stress" imaging one hour after initiation of the patients exercise session. While imaging one hour after exercise is clearly not a period of time when the individual is "stressed", the terminology persisted since this image was the first image obtained following the stress. Subsequently, these images became known as "stress" images. Over time, thallium distribution changed and consequently the appearance of the images themselves changed. This became known as "redistribution" since the distribution of Th201 changed with time. For lack of a better term, this second image was coined a "resting" image and their comparison was used to define changes, which were thought to represent ischemia and possibly infarction. As we shall see in subsequent sections, neither Blumgart (1926) nor Gorlin (1959) considered this to be so and a better understanding of their work might have saved Nuclear Cardiology decades of misdirection. However, the actual ability to detect infarction was not through the rest image obtained in this manner; but rather, a resting (Parkey 1976, Ahmad 1979, Walsh 1977) image obtained by using Tc-99m pyrophosphate (Tc-99 PYP), which required imaging of the heart within 48-72 hours of the time of infarction to accurately detect. Efforts to replace Tc-99m PYP with newer imaging agents (Khaw 1999) not only emphasize the limitations of timing and possible overlap of bone activity; but, the importance of utilization of resting images and not stress images to determine the presence or absence of damaged (infarction, stunned, hibernating) myocardium, as emphasized by Blumgart (1926) and Gorlin (1959).

Over time, the lessons of Blumgart and Gorlin were all but forgotten and the terms "stress" and "rest" anthropomorphically took on a life and meaning of their own. Instead of viewing comparative Nuclear images obtained under same state conditions at two different points in time, which could then be compared to look for changes in distribution of isotope scintillation consistent with changes in supply (blood flow) and function (cellular, including mitochondria), the introduction of technetium- $99 \mathrm{~m}$ isotopes were introduced with directions by the manufacturers that clinicians and scientists needed to give two injections (rest stress), instead of one (stress-redistribution) used for Tl-201. In the first instance, teboroxime (Fleming 1991a) was reported as having too rapid a "washout" (another term for redistribution), which would prevent multiple imaging given the camera acquisition speed of the time, while Sestamibi would be promoted as having no washout allowing one to chose when they wanted to image the patient; a property which would be warmly welcomed by nuclear medicine departments concerned with the number of studies being ordered and nuclear departments scheduling issues. Truly, this alleged property of Sestamibi was a welcomed relief for such scheduling issues. This misinformation continued to drive the market reducing study accuracy despite multiple studies reporting that Sestamibi did in fact redistribute. By using this redistribution property, investigators have been better able to define heart failure (Hurwitz 1993, 1998, Saha 1994, Giubbini 1995, 
Sugiura 2006, Kumita 2002), cardiomyopathy (Matsuo 2007, Ikawa 2007, Meissner 2002) and coronary artery vasospasm (Ono 2002, 2003). Perhaps the most compelling work was published by Maublant (1988), prior to the introduction of Sestamibi into the United States. This work revealed that Sestamibi has a 28 minute washout under non-ischemic conditions while the work of Crane (1993), which was funded by the pharmaceutical company itself, demonstrated that under ischemic conditions, the washout of Sestamibi occurred more rapidly due to mitochondrial calcium overload. This information, to date, continues to appear within package inserts as shown in Figure 1 and is now described as having "minimal cardiac redistribution," encouraging physicians to use multiple doses for studies.

REST

STRESS

\begin{tabular}{lcccccccc}
\hline & \multicolumn{2}{c}{ Heart } & \multicolumn{2}{c}{ Liver } & \multicolumn{2}{c}{ Heart } & \multicolumn{2}{c}{ Liver } \\
\cline { 2 - 8 } Time & Biological & Effective & Biological & Effective & Biological & Effective & Biological Effective \\
\hline 5 min. & 1.2 & 1.2 & 19.6 & 19.4 & 1.5 & 1.5 & 5.0 & 5.8 \\
30 min. & 1.1 & 1.0 & 12.2 & 11.5 & 1.4 & 1.3 & 4.5 & 4.2 \\
1 hour & 1.0 & 0.9 & 5.6 & 5.0 & 1.4 & 1.2 & 2.4 & 2.1 \\
2 hours & 1.0 & 0.8 & 2.2 & 1.7 & 1.2 & 1.0 & 0.9 & 0.7 \\
4 hours & 0.8 & 0.5 & 0.7 & 0.4 & 1.0 & 0.6 & 0.3 & 0.2 \\
\hline
\end{tabular}

A study in a dog myocardial ischemia model reported that Technetium TC99m Sestamibi undergoes myocardial distribuion (redistribution), although more slowly and less completely than thalous chloride Tl-201. A study in a dog myocardial infarction model reported that the drug showed no redistribution of any consequence. Definitive human studies to demonstrate possible redistribution have not been reported. In patients with documented myocardial infarction, imaging revealed the infarct up to four hours post dose.

Fig. 1. Cardiolite US PI 513121-0309 (3-16-2009 package insert)

After our initial work (Fleming 1991a) with these Tc-99m isotopes in 1989, we published several papers (Fleming 1995, 1998, 1999a-b, 1992a-b, 2000a-b, 2002a-b) under the misinformation that two injections of these compounds were required and like many others we failed to fully understand the work of Blumgart and Gorlin. By 1999-2000, Sestamibi was being used for the detection of breast cancer. The company involved with this work (under the trade name Miraluma) was concerned that clinicians were not ordering the study due to what was perceived as incorrect results. Studies performed show "abnormal" results without the detection of biopsy proven breast cancer. However, the tissue while not cancerous was not "normal" either and considerable confusion existed at the time. We were asked to conduct breast imaging studies and developed a method for simultaneous detection of both heart disease and breast disease. The results were published (Fleming 2002c-e, 2003a-c) and presented at several meetings as shown in Figure 2. While beyond the scope of this text, it resulted in our looking more closely at the information, which the nuclear computer was seeing (Fleming 2003d) and which we were not; viz. the actual scintigraphic counts from regions of interest (ROIs) as shown in Figure 3 for the breast and Figure 4 for the heart. This approach not only gave us a greater insight as to the cause of angina, viz. regional blood flow differences (Fleming 2003d); but, it allowed us to additionally see for the first time detectable evidence of an activated thymus gland (Fleming 2003e) with enlargement and increased isotope uptake associated with coronary artery disease/inflammation (Fleming 1999c, 2003e, 2008a) and providing further evidence of underlying coronary artery disease. This same state physiologic approach allowed us to compare images of the heart taken at 5 minutes and 60 minutes following pharmacologic 
stressing of the heart utilizing a single injection of the isotope Sestamibi. These rather crude early findings (Figure 5) clearly demonstrated that individuals with ischemic heart disease revealed different distribution patterns of Sestamibi within cardiac tissue at 5 and 60 minutes post stress, proving that Sestamibi in fact "redistributes" within the heart and confirmed the information reported by others as noted above and as we will see in the remainder of this chapter, it has been confirmed by others world wide as well as recent work out of both UCLA and Harvard (Sheikine 2010). We have also demonstrated (Fleming 2009) that technetium-99m tetrofosmin (trade name Myoview) similarly redistributes. Since it is imperative that comparison images be performed on the same SPECT camera to allow meaningful comparisons of the two images, we will now turn our attention to the details of when nuclear SPECT cameras provide us the information we are seeking and when they do not.

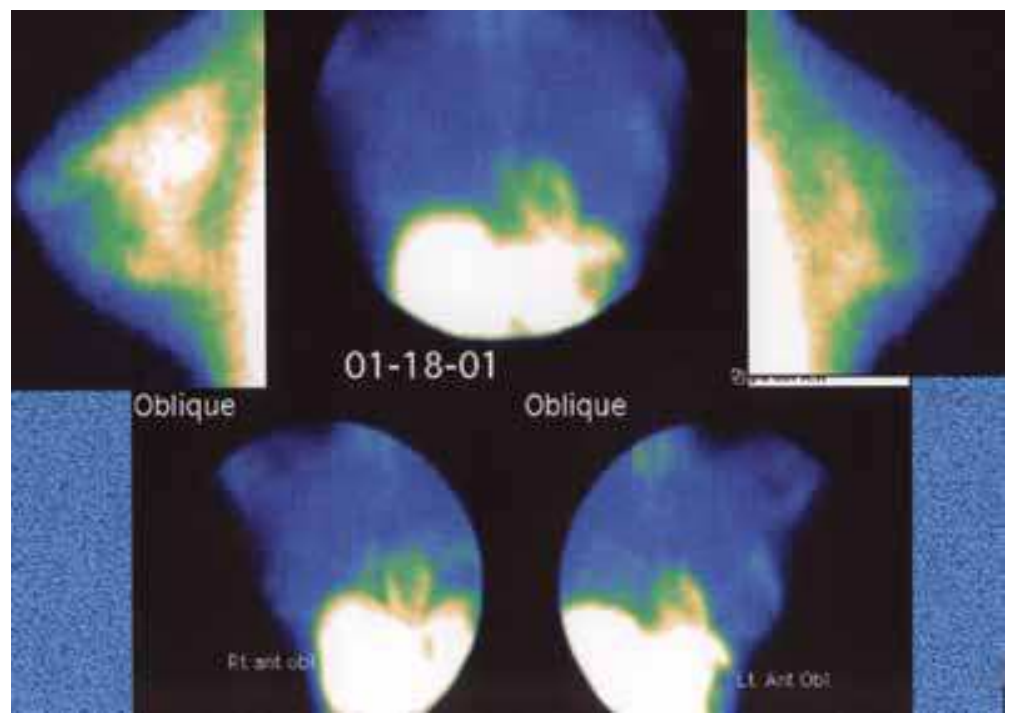

Fig. 2. Acquisition of Heart and Breast Image 5 Minutes After Stress Injection

Information obtained from the package insert of this product reports that "Definitive human studies to demonstrate possible redistribution have not been reported." The information provided as discussed in the text and literature clearly shows this not to be the case.

Following pharmacologic stress and injection of sestamibi, images of cardiac and breast tissue were acquired using a SPECT camera 5-minutes after cessation of stress. Detection of thymic tissue, while not noted in this individual, has been published by us elsewhere (Fleming 2003e).

Here breast enhanced scintigraphy test (BEST) images from three different women are quantified using regions of interest (ROIs) and compared with results (immediately below each image) obtained by Dr. William Dooley using ductoscopy. The results of tissue specimens were confirmed pathologically as representing inflammatory tissue and cancer respectively as shown in the images. This study provided further information as to the ability to reliable "quantify" differences on Nuclear imaging using ROI and the role of scintigraphy measurement in diagnosing both heart disease and breast cancer. 

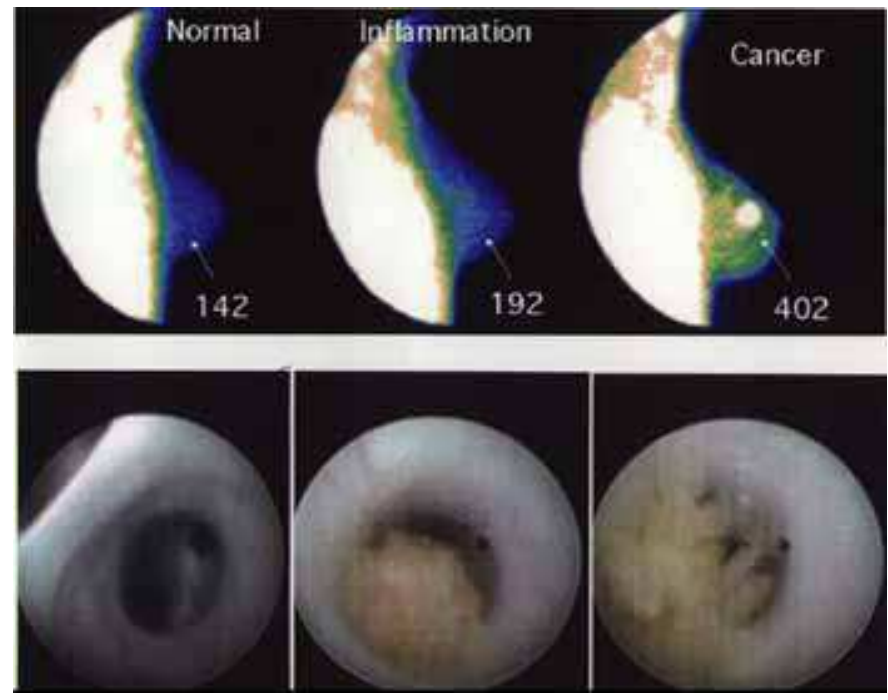

Fig. 3. Analysis of regions of interest (ROIs) of breast tissue

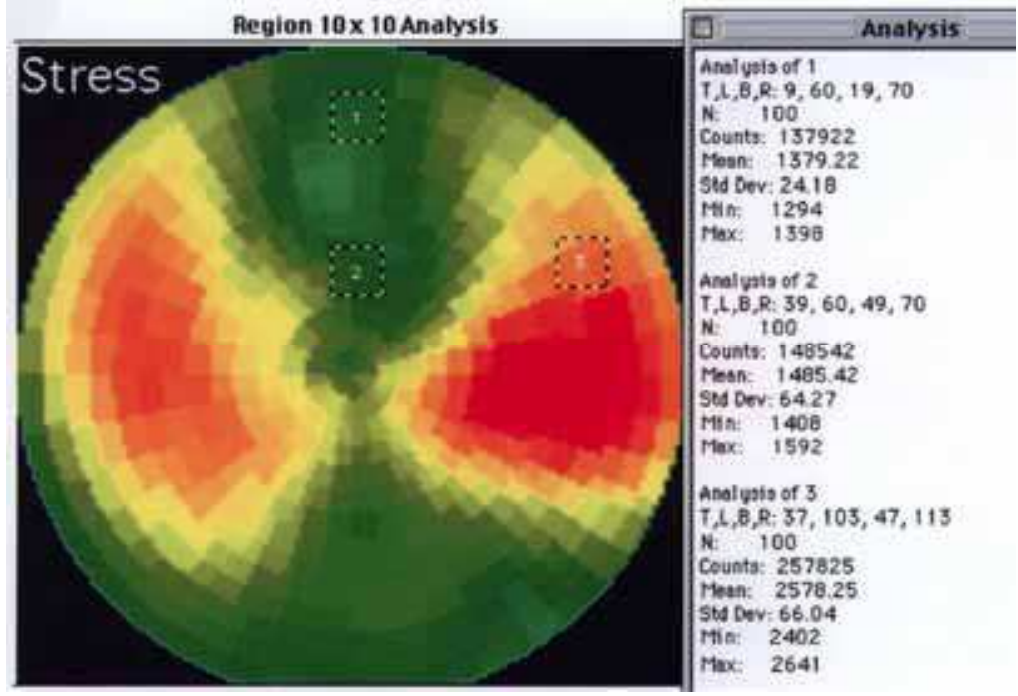

Fig. 4. Analysis of regions of interest (ROIs) from Cardiac bullseye images

While qualitative Gestalt of images are frequently utilized for both clinical and scientific work, regions 1 (mean count of 1379.22) and 2 (mean count of 1485.42) represents an almost $8 \%$ difference in count activity which cannot be appreciated visually. The quantification of the differences in regions (ROIs) of interest (Fleming 2003d) was utilized to demonstrate that angina is not the result of narrowed arteries; but rather, the reduced ability of diseased arteries to vasodilate to the extent that non-diseased coronary arteries can dilate (viz. regional blood flow differences) under the influence of pharmacologic stress. 
a) 5 Minute post-stress image taken on NucLear MAC SPECT Camera

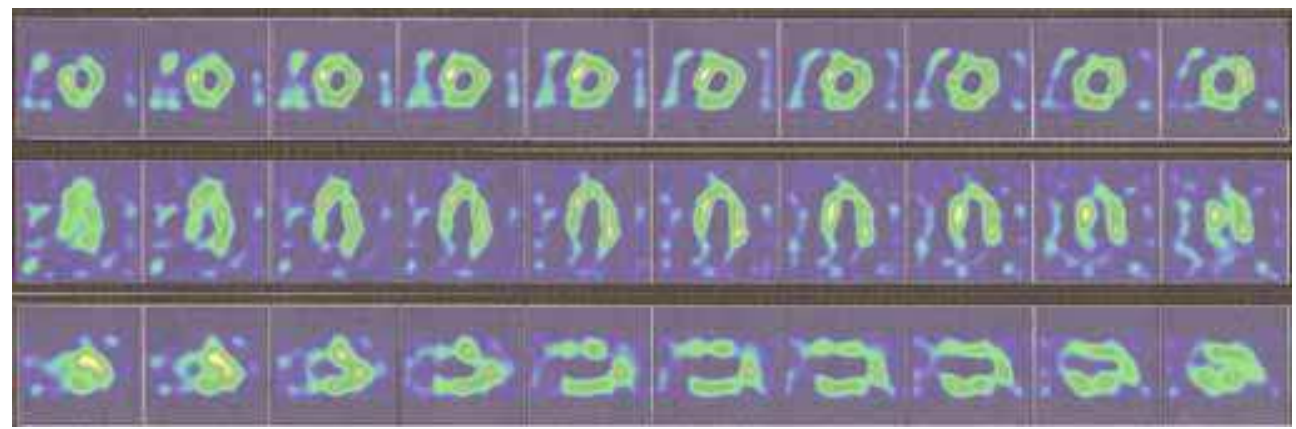

b) 60 Minute post-stress image taken on NucLear MAC SPECT Camera

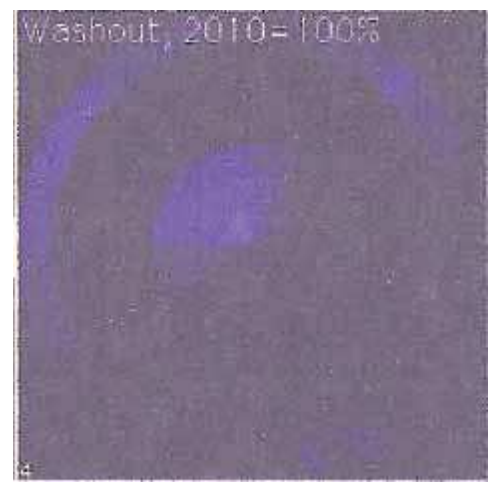

c) Washout of Sestamibi as determined by NucLear MAC Spect Camera system

Fig. 5a-c. Early comparisons of coronary artery disease showing differences in Sestamibi distribution at 5 and 60 minutes post stressing of the heart

This early study SPECT camera image reveals an anterior image of the heart acquired 5 minutes after peak cardiac stress and Sestamibi injection, demonstrating no regional defects in isotope activity.

This qualitative study, performed in 2001 of the same patient in figure 5A, shows washout of Sestamibi compared with the 5-minute image. Here there are both distal anterior and apical reductions in isotope in addition to a dilated right ventricle. Clearly, these early 2001 studies 
demonstrated that Sestamibi redistributes throughout cardiac tissue with different results seen at 5 and 60 minutes post-stress. This is confirmed in the "washout" study seen in Figure 5C. Bullseye definition of "washout" made by the same SPECT camera system using it's computer software to compare the two sets of images shown above (5A and 5B). The results show a qualitative (blue) redistribution/washout in the distal anterior and apical regions. Clearly, these 2001 images show differences in isotope distribution between the 5 and 60minute images. Similar findings have now been reported by others, including researchers at UCLA and Harvard Universities (Sheikine 2010).

\section{The Fleming-Harrington nuclear imaging uncertainty principle. Understanding the physics of how nuclear SPECT cameras really work?}

Abstract: The introduction of the Heisenberg Uncertainty principle and Nuclear Cardiology occurred simultaneously in 1925-1927. Thirty years later the Anger gamma camera would allow for a more sophisticated radioactive isotope counting to determine the presence or absence of disease. When employed with technetium- $99 \mathrm{~m}$ isotopes, ischemic heart disease can be inferred by differences in visual appearance of cardiac images. These gestalts of imaging results have been separated from the quantitative information recorded by the cameras computer. We investigated whether current camera and computer systems are sophisticated enough to quantify differences between images to be clinically relevant. Our study demonstrated that efforts to "sharpen" image appearance does so at a reduction in "accuracy". Like Heisenberg, this work shows that one cannot know the exact location and the amount of activity simultaneously and that a decision must be made for accuracy over image sharpness if one is to truly quantify differences in isotope concentration between images.

In 1927, Werner Heisenberg (Heisenberg 1927), published his "Uncertainty principle" which in brief states that it is impossible to simultaneously know both the position and momentum of an electron or any other particle with any degree of accuracy or "certainty." To define the location of an electron required interaction with it. This interaction would result in movement of the electron and as a result, would move the electron. The best one could achieve is knowledge of where the electron was at the time of interaction.

The first utilization of nuclear isotopes for medical imaging and evaluation of heart disease was conducted by Blumgart (1926) in February of 1925 when he injected himself with Radium C, which emits alpha and beta particles and gamma rays. As such, the Geiger counter chamber developed by Blumgart and Yens, could detect the passage of blood carrying the radium. The studies would first be published in 1926 (Blumgart 1926) and become known as "circulation time" and would dynamically define myocardial contractility by comparing changes in count activity over time.

In 1957, Hal Anger (1957) demonstrated the first gamma camera designed to detect the emission of radioactive decay emanating from the patient. In essence, a modern Geiger counter which could be held some distance from the chest to measure isotope decay while present in cardiac tissue. The decay (scintillation) is detected by the camera after being absorbed by the camera crystal (usually sodium iodide) with the subsequent release of an electron from the sodium iodide, which is subsequently detected by a photomultiplier (PMT) tube as shown in Figure 6. These scintillations are tallied by the computer. These anger cameras have been used to image various regions of the body using radioactive isotopes which are known to localize to the tissues in question. For cardiac disease this has primarily included thallium-201 and technetium-99m. 
The utilization of these cameras have been assumed to be able to detect changes in isotope availability by "counting" the amount of radioactivity as described and translating this information into a black and white or color format image for human viewing and interpretation of disease. However, to the best our knowledge, no such experimentation has been carried out to determine if today's gamma cameras can in fact accurately count radioactive decay required for image comparison. This quantification is necessary to compare redistribution of isotopes and to avoid errors in interpretation. This requires more than simply the ability to produce pictures of different brightness; it requires actual ability to measure differences in isotope decay (scintillation), as did Blumgarts Geiger counter. This is the basis of devices used to determine human radiation exposure and is a requirement in diagnostic imaging. To investigate this we conducted a series of experiments with known quantities of Tc-99m and utilized two different matrixes commonly employed in clinical cardiology to determine if the cameras are able to accurately measure radioactive decay.

The acquisition of technetium-99m isotopes for diagnostic purposes results from the radioactive decay of the parent compound (Molybdenum) to the daughter compound (technetium-99m) is shown in Figure 7. The half-life for technetium-99m is 6.01 hours as shown in Figure 8. Each sample of technetium-99m contained $10.1 \mathrm{mCi}(37.37 \mathrm{MBq})$ of radioactive compound. Each sample was sealed in a syringe preventing any leakage of material. If the cameras are able to accurately measure isotope decay, the camera should reveal a $10 \%$ reduction in count activity from the first image and the second image taken 55 minutes later. Utilizing a Philips Forte Dual head single photon emission computed tomography (SPECT) camera with general all purpose collimators, the camera was set to (1) a 64 × 64 matrix and (2) $128 \times 128$ matrix settings. As shown in Figure 9A-C, the greater the matrix (pixel) settings per image, the greater the localization of isotope emission within the field of view and the sharper the image. However, for each pixel, there are surrounding septa of lost information forming the border of the pixel. The question is whether the increase in image "sharpness" comes at the expense of "accuracy" of radioactive isotope count activity, which is the basis for image comparisons.

As shown in Figure 10A, the initial radioactive count obtained over 5 minutes from a syringe of $37.37 \mathrm{MBq}$ of radioactive tc- $99 \mathrm{~m}$ using a 64 by 64 pixel matrix was 1,405,721. Using the same matrix and imaging 55 minutes later, Figure 10B shows the counts collected over 5 minutes were 1,251,359. The decay curve for tc- $99 \mathrm{~m}$ means that there should have been a $10 \%$ reduction in count activity. In this instance, there was a $10.98 \%$ reduction in measured isotope activity. When efforts to improve image "sharpness" were made by changing the matrix to $128 \times 128$, Figure 10C shows the initial count activity measured over 5 minutes was $3,473,001$. When the syringe was reimaged 55 minutes later using the $128 \mathrm{x}$ 128 matrix, the counts collected over a 5 minute period, as shown in Figure 10D, were $2,966,394$. While there was an increase in actual radioactive count activity compared with the $64 \times 64$ matrix, this came at a cost of accuracy with a $14.59 \%$ reduction in radioactive count activity. This is 4.68 times the data lost as was seen with the $64 \times 64$ matrix.

While the visual appearance desired by most clinicians to make diagnostic decisions is important, images can be manipulated to confirm what the diagnostician is looking for. These adjustments may lead to incorrect conclusions and visual interpretations alone may lead to incorrect conclusions. When done at social gatherings, these visual illusions as shown in Figure 11 can be entertaining. When done to determine if someone has ischemic heart disease, such illusions can be more distressing. For that reason, multiple researchers have been trying to develop algorithms, which will reduce this human error. It is impossible to reduce this human error, if Section of the error is the result of instrumentation. 
These findings have demonstrated that independent of the visual image seen by the clinician, this information is dependent upon the accuracy of the computers ability to detect the radioactive decay of isotope needed to make image comparisons. Like, Heisenberg's uncertainty principle, this uncertainty principle comes at a cost. The ability to detect the location of the emission of the gamma ray is influenced by the matrix surrounding the acquired image of the heart. This precision of location comes at the cost of lost accuracy as to the number of gamma rays being emitted. Given this spatial limitation (sharpness) versus accuracy, like Heisenberg we are left with a dilemma. Do we sacrifice accuracy for "sharpness" or should we be more concerned with the necessary accuracy required to compare images (Fleming 2009b, 2010a-b, Sheikine 2010), thereby reducing errors made in evaluation of the extent of ischemic heart disease?

Now that we have defined the limitations and accuracy of our SPECT cameras to define the presence or absence of disease, we can now turn our attention to understanding the kinetics of nuclear isotopes, with particular attention to technetium-99m compounds (viz. Sestamibi) to better understand how to best study the heart using our SPECT cameras.

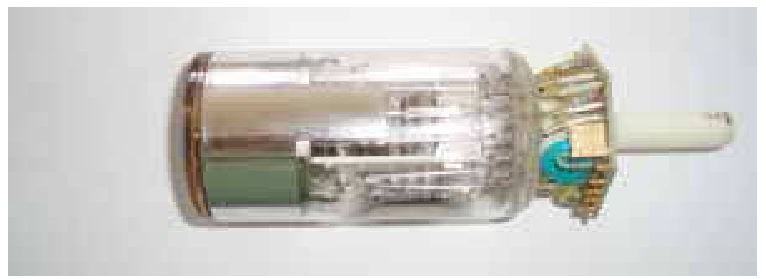

Fig. 6. Photomultiplier (PMTs) Tube

As shown, PMTs are composed of glass tube with a vacuum inside. Photons leaving the patient would approach the PMT from left to right, striking the photocathode material first. This results in electrons being produced as a consequence of the photoelectric effect. The focusing electrode subsequently directs these toward the electron multiplier composed of a series of electrodes (dynodes), each with a more positive voltage than the next. The electrons are accelerated toward the first dynode, arriving with a greater energy. This results in low energy electrons being generated by the first dynode, which are in turn accelerated toward the second dynode and so on. The process is known as secondary emission and results in an amplification of the original scintillation. The electrons finally reach the anode (far right Section of PMT) where the accumulated charge results in a sharp current pulse indicating the arrival of the photon at the photocathode.
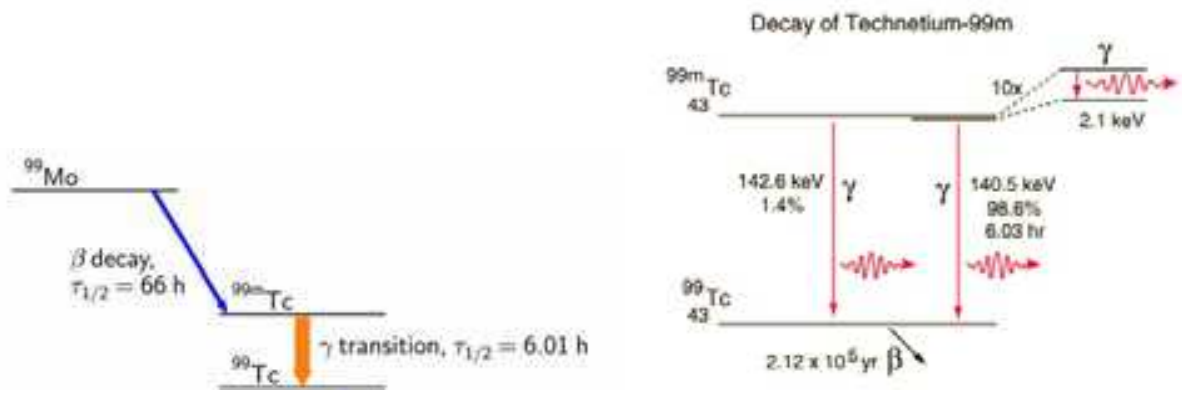

Fig. 7. Decay of Technetium-99m 
The production of technetium by bombardment of a molybdenum atom with deuterons was first documented by Carol Perrier and Emilio Segre in 1937. Technetium-99m (meta stable) decays to Technetium 99 through the release of a gamma (photons) rays of $140.5 \mathrm{keV}(98.6 \%)$ and $142.6 \mathrm{keV}(1.4 \%)$ as shown. The final result is Technetium-99 (Tc-99) with a half-life of 210,000 years.

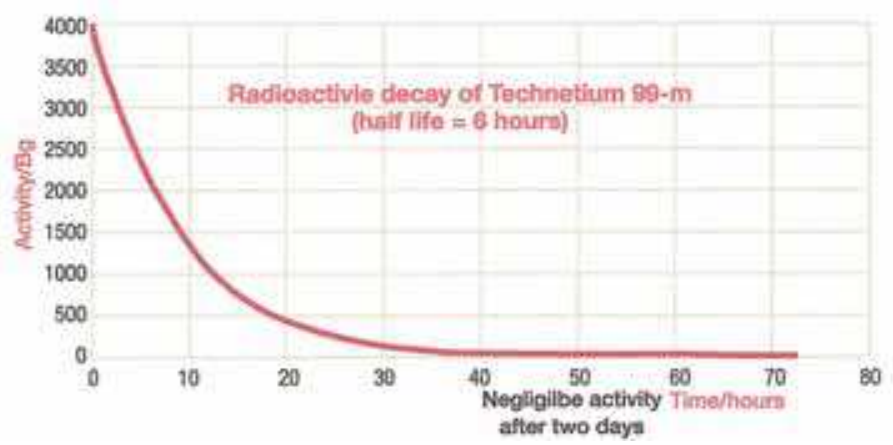

Fig. 8. Radioactive decay curve of Technetium-99m

The radioactive decay of technetium- $99 \mathrm{~m}$ is shown. The physical half-life for Tc- $99 \mathrm{~m}$ is 6.01 hours. Given this information, one can calculate that over the course of 55 minutes there is a $10 \%$ decay of the isotope. In clinical studies looking at sestamibi redistribution to determine ischemia (Fleming 2009b, 2010a) the initial stress imaging is made at 5 minutes with the second imaging at 60 minutes.

An effort to improve image "sharpness" (Figure 9A) is the result of increasing the number of pixels in a given field. Cameras set up with a $64 \times 64$ matrix (pixel) resolution, will result in a more blurred image, while a matrix of 128 x 128 (pixels) will increase image "sharpness." As seen in Figures 9B and 9C, the cost of increasing "sharpness" occurs at the cost of more septa separating each pixel, which reduces the area available for information acquisition. Each septa itself is excluded from acquiring information on radioactive decay, exchanging information for sharpness.

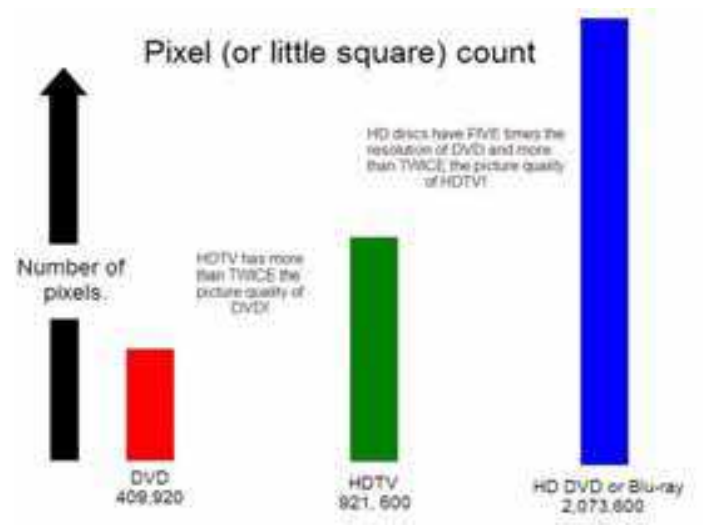

Fig. 9a. Increasing the number of pixels increases image "sharpness" 
Fleming-Harrington Redistribution Wash-in Washout (FHRWW):

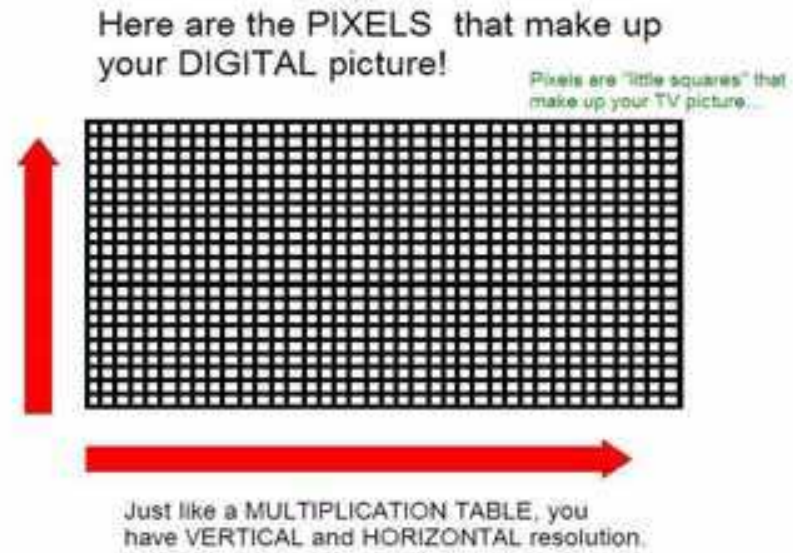

Fig. 9b. Fewer pixels reduce "sharpness" while increasing scintillation detection

SMALLER pixels means MORE pixels. MORE pixels means a SHARPER picturet

FYI RESOLUTION MEANS PICTURE OUALITYI

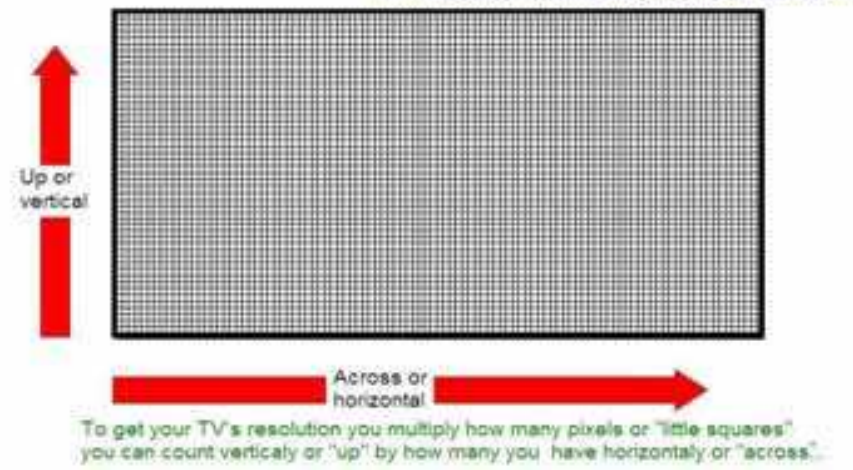

Fig. 9c. More pixels increase image "sharpness" but at the expense of scintillation detection Fig. 9a-c. The greater the number of pixels, the "sharper" the image "appears"

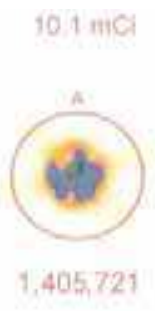

a)

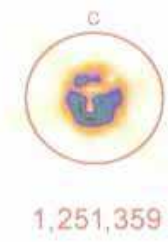

b)

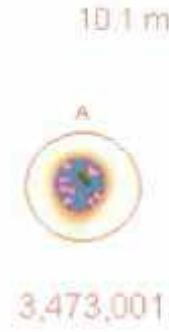

c)

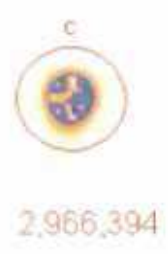

d)

Fig. 10. Verification of Technetium-99m-Sestamibi Count Imaging 
A $37.37 \mathrm{MBq}$ syringe of technetium- $99 \mathrm{~m}$-sestamibi is placed under the camera and counts acquired over 5 minutes. The same syringe is reimaged 55 minutes later for an equal amount of time. When the $64 \times 64$ matrix was used, the original count was 1,405,721, the second image count was $1,251,359$, representing $89 \%$ of the original count activity. When the resolution was increased to $128 \times 128$ matrix, there was an additional 50\% loss in data, with a 5-minute image (different syringe sample) count of $3,473,001$. The second image count 55 minutes later was $2,966,394$, representing a count decrease of $14.6 \%, 4.6 \%$ more than should have been shown. This reduction is due to data lost as a result of increased grid lines forming the increased number of pixels per image. Subsequently, total information is lost at the gain of localization information (FH Uncertainty principle). Since we are looking for total cell uptake of the tracer, one must use the $64 \times 64$ matrix as a better index of isotope activity in exchange for resolution.

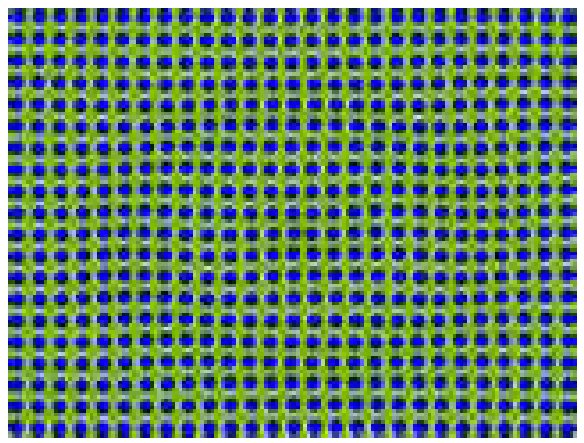

Fig. 11. The importance of recognizing visual illusions

Visual illusions such as this shown here can result in incorrect diagnostic decisions when depended upon by the clinician. This particular illusion demonstrates visual problems resulting from pixel information. When these illusions are the result of instrumentality, diagnosticians cannot reliably use them to make clinical decisions and the utilization of attenuation algorithms cannot reliably reduce these errors, making it even more important that we know what our nuclear cameras are truly capable of measuring and how to most accurately use them.

\section{The kinetics of radioactive isotope distribution from vein to heart to cells to redistribution}

Imaging protocols are frequently employed by clinicians with an incomplete understanding of the kinetics of a given isotope. Consequently, patients receive more isotope than may be necessary and under the wrong conditions for detection of the question at hand. E.g. the utilization of stress-rest OR rest-stress imaging, frequently results in two injections of radioactive isotope, when only one is needed, resulting in comparing apples and oranges. Resting images as discussed elsewhere in this chapter are useful only for determining tissue viability and do not augment coronary artery blood flow (viz. coronary or stenosis flow reserve) as discussed in Figure 14 below and elsewhere in this chapter and as such cannot be used to determine the presence or absence of ischemia. Similarly, resting images are not helpful for comparison with stress images as discussed in Section IV of this chapter and results in failure to accurately detect ischemia along with assignment of ischemia to the wrong regions of myocardium, leading to continued frustration for those using stress-rest 
protocols. In this Section of the chapter, we will review the process by which nuclear isotopes are injected, delivered and redistributed throughout the myocardium allowing us to better understand how we can take advantage of these properties to increase our detection of ischemia and as we shall see in Section $\mathrm{V}$, the ability to detect myocardial viability.

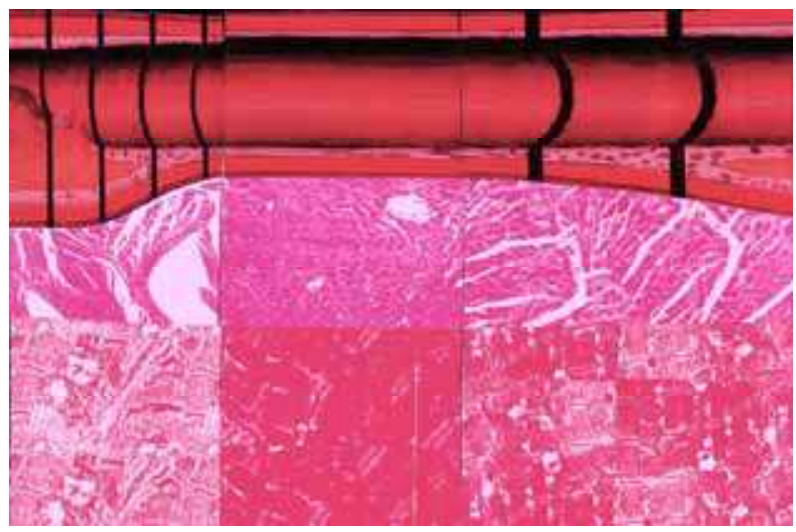

Fig. 12. Diagram of coronary artery prior to injection of radioactive isotope

Figure 12 represents an artery with the lumen of the artery at the top of the image. On the left side is a critically narrowed artery with a vulnerable plaque, the region immediately below that represents myocytes when ischemic as seen under the microscope. Immediately below this is the appearance of mitochondria under severe ischemic conditions. Note the reduction in myofibrils and mitochondria under severe ischemic conditions. In the middle third of the image, at the top, lies a normal appearing coronary lumen with normal appearing myocyte with normal concentration of mitochondria immediately below the myocytes image. The right Section of the image represents diseased artery initially represented by extension outward as first demonstrated by Glagov (1987) from pathology specimens with encroachment into the lumen as we proceed from left to right. Immediately below the artery is the microscopic appearance of mildly damaged myocytes with the lower Section of the image revealing a reduction in the numbers of mitochondria present in regions with mild to moderate ischemia, but, more than the region of vulnerable plaque (left side of image) where severe ischemia is present.

Figure 13 shows the longitudinal and cross-sectional effects of an artery without disease on the left and a region of artery with inflammatory coronary artery disease resulting in ischemia. Arteries free of disease can vasodilate upon command to increase coronary blood flow while arteries with inflammation are unable to dilate to the same degree. These differences can be detected and measured in the nuclear laboratory as we have shown (Fleming2008a).

Figure 14 shows the relationship between coronary (stenosis) flow reserve and percent diameter narrowing. As established by Gould (1990) in dogs and in humans by Fleming $(1991 b, 1994)$ the greater the amount of inflammatory coronary artery disease (ischemia), the less the flow reserve and the greater the reduction in an arteries ability to carry blood flow along with radioactive isotopes to a region of myocardium where the isotope can cross the capillary membrane and migrate into myocytes for uptake and detection. These differences in flow were first established by Poiseuille (1840) in the laboratory. 


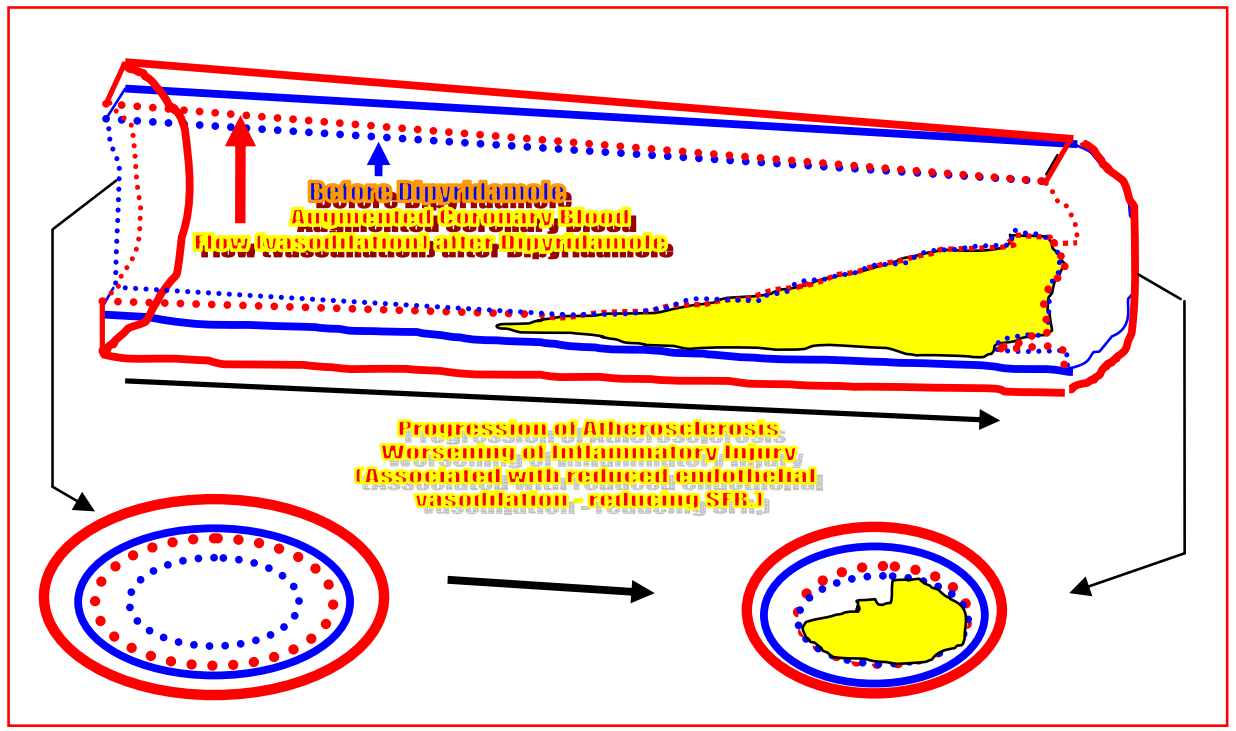

Fig. 13. Demonstrates the capability of coronary arteries to dilate to increase coronary blood flow, viz. stenosis (or coronary) flow reserve, to meet increased myocardial oxygen and nutrient demands with "stress"

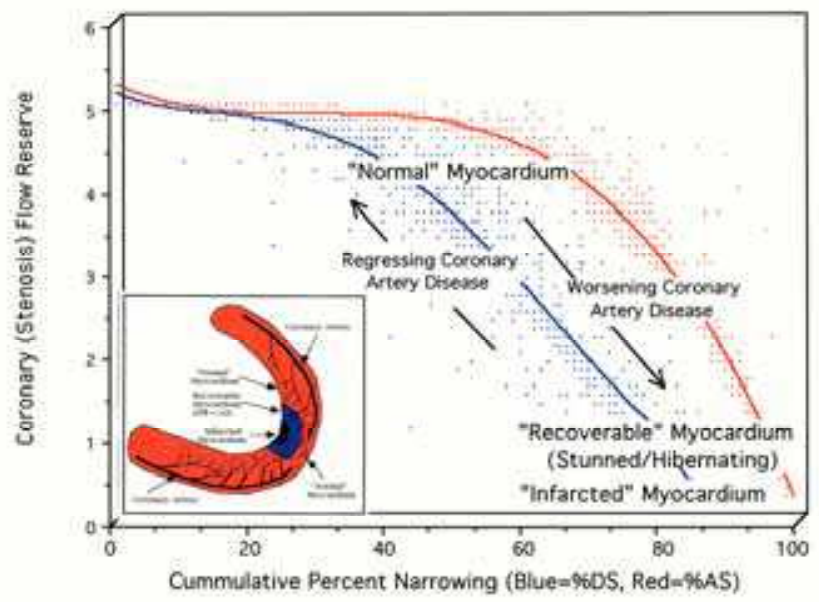

Fig. 14. Actual coronary flow reserve in humans as influenced by anatomic narrowing of coronary arteries

Figure 15 shows the injection of sestamibi into the right antecubital region immediately following stress. The radioactive material can be measured as documented by Blumgart (1926) to determine circulation time and myocardial status.

Figure 16 shows the same artery from Figure 12, with the initial pass of radioactive isotope material through the lumen. 
Fleming-Harrington Redistribution Wash-in Washout (FHRWW):

The Platinum Standard for Nuclear Cardiology

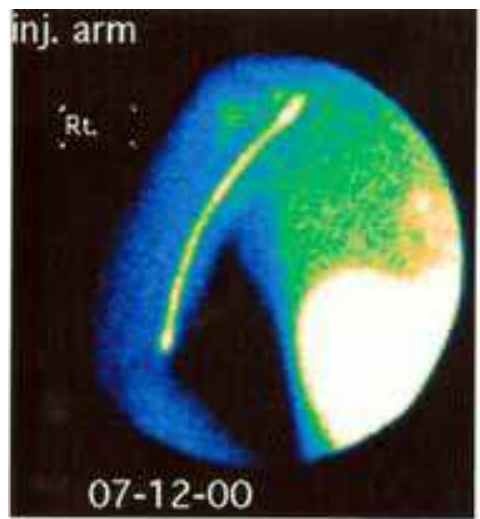

Fig. 15. Initial injection of technetium-99m into the right antecubital vein

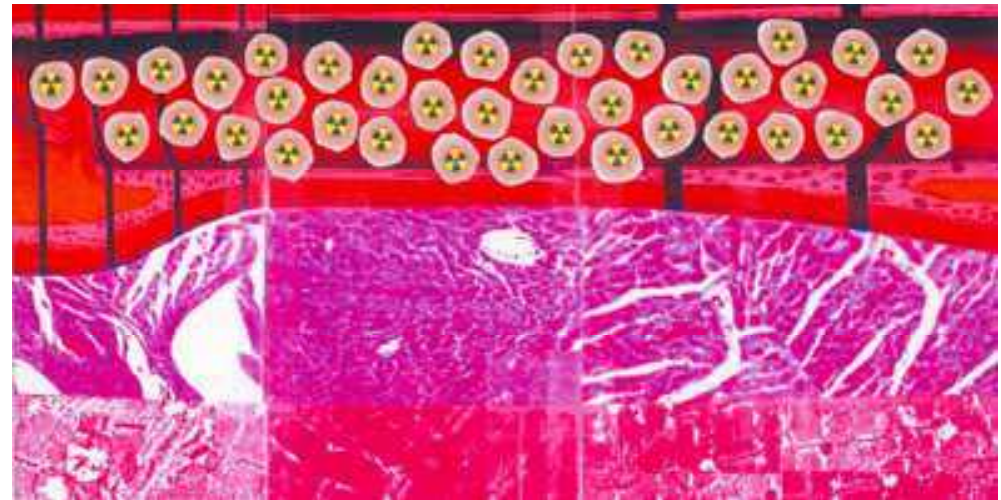

Fig. 16. Cartoon depiction of radioactive isotope initially entering a coronary artery.

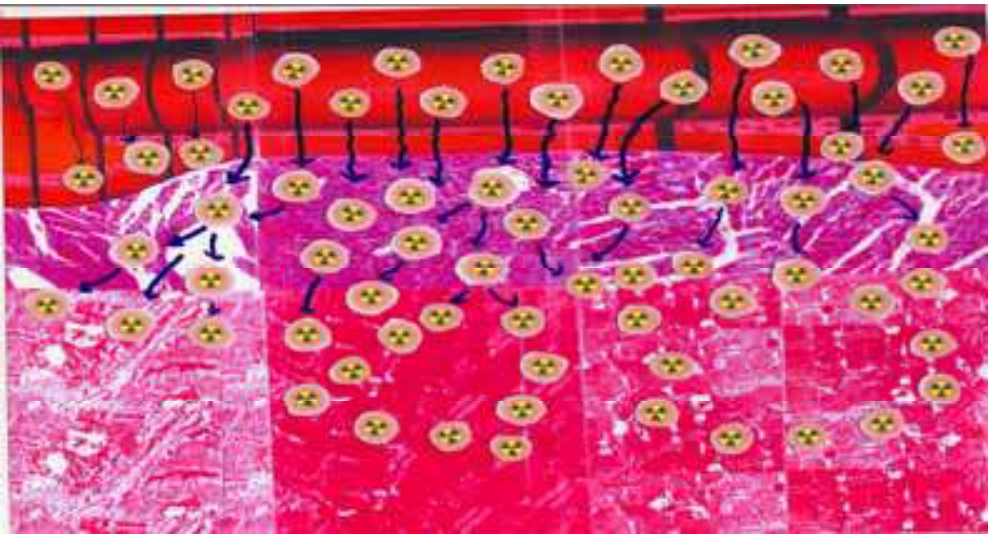

Fig. 17. Cartoon depiction of delivery of radioactive compound from a coronary artery into the myocardium 
This figure shows the passage of radioactive isotope (sestamibi) from the lumen into the myocardial tissues 5 minutes after injection into the venous system following "stress". Passage from lumen to tissue is dependent upon four variables. First, when there is a region of artery is inflamed, there is (a) reduced passage of material through the region from the narrowing as well as a reduction in the dilatation of the artery to carry radioactive material as described in Figure 14. Secondly, as the wall of the artery is inflamed and thickened, there is an increased impediment to the movement of sestamibi through such a region in contrast to a non-inflamed region. Thirdly, as a region becomes ischemic, the mitochondria are less able to retain the sestamibi due to calcium overload (Crane 1993, Lenzi 2003, Kodavanti 2008) and there are fewer mitochondria to retain the sestamibi. Finally, sestamibi is a lipophilic compound (Maublant 1988) which when passing through (a) a lipid laden plaque and (b) an inflamed region or region with increased mitochondrial activity (Fleming 2000c) due to the white blood cells (greater mitochondrial concentration is required of these cells if they are to be able to respond to inflammatory stimuli including diseased arteries or bacteria which may or may not play a role in a disease artery) will have a greater uptake (Fleming 2002c-e, 2003a-c) of sestamibi, reducing the amount of radioactive tracer passing through the lumen into myocardial tissue with its mitochondria.
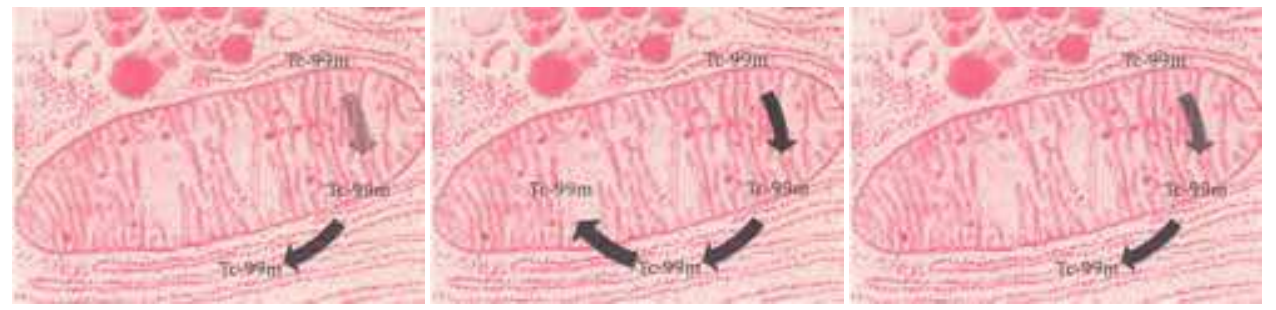

Fig. 18. Electron microscopic images of mitochondria showing the outer and inner membranes along with cristae and matrix

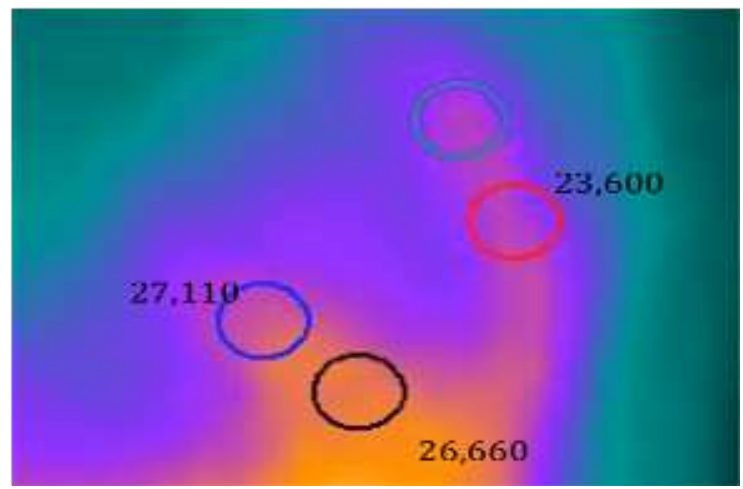

Fig. 19. SPECT image of distribution of Sestamibi within myocardial tissue which can be qualitatively Gestalted or quantitatively measured as done here

Figure 18 shows three mitochondria under different sets of conditions, as shown in Figure 17. The far left mitochondria shows the reduced delivery of sestamibi in a region of vulnerable plaque where the reduced lumen size, reduced flow reserve ability, the increased 
lipid laden material will result in a reduction in delivery of sestamibi to the mitochondria in the region. The middle panel shows a mitochondria receiving maximum delivery of sestamibi from an artery, which is able to vasodilate and carry maximum amount of isotope with no impedance to delivery of the isotope to the mitochondria and non-ischemic mitochondria. The far right panel shows more sestamibi delivery than the region with vulnerable plaque, with reductions due to limitations in lumen size and reduced flow reserve, but, not as limited as that occurring with the region of vulnerable plaque.

Figure 19 shows an actual myocardial perfusion image obtained 5 minutes after injection of sestamibi with three regions of interest (ROIs) measured in regions of (a) a vulnerable plaque $(23,600),(b)$ a normal region $(27,110)$ and finally (c) a region with ischemia without critical narrowing or vulnerable plaque $(26,660)$.

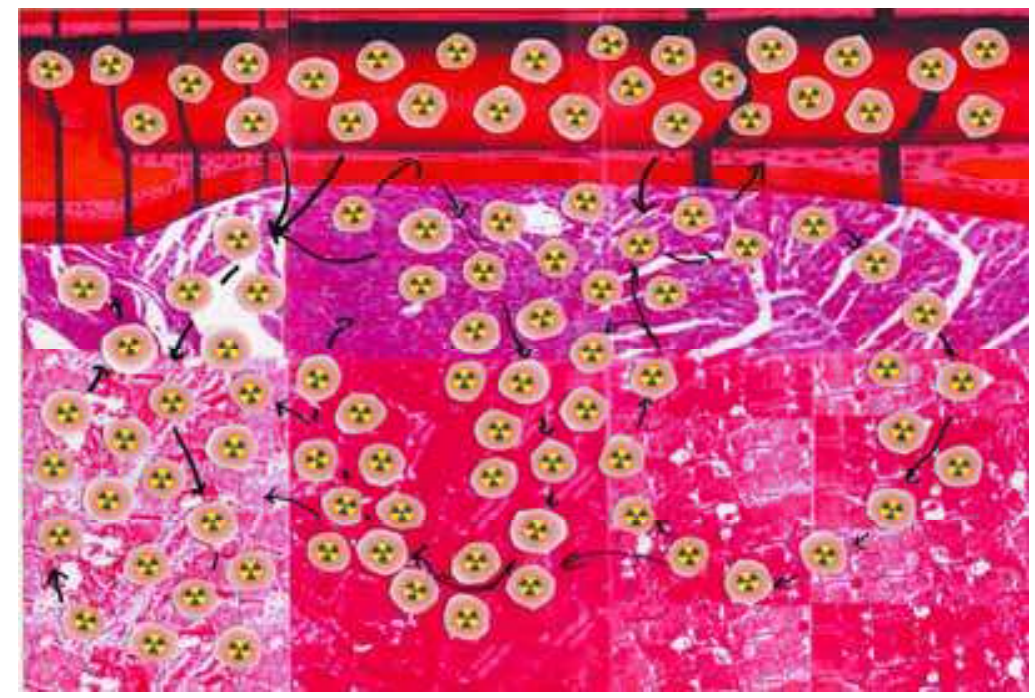

Fig. 20. Coronary artery cartoon showing redistribution of radioactive isotope (in this instance sestamibi) with time

Figure 20 shows the effect of 55 additional minutes (60 minutes post-stress) of perfusion of tissue with sestamibi. During this time, there has been additional delivery of sestamibi to each of the regions of the heart. As established by Maublant (1988), the washout for sestamibi is 28 minutes. Crane(1993) established that ischemia affects the calcium concentration within mitochondria resulting in calcium overload and decreased potentiation for sestamibi retention. Between these two factors in addition to the four reasons discussed with Figure 17, regions of critically narrowed arteries or arteries with vulnerable plaque, as shown in the left side of the figure will have an increase in radioactive (sestamibi) count (concentration) at 60 minutes compared with that seen at 5 minutes. In the middle of the figure, the region of myocardium supplied with a normally functioning artery without narrowing sees a constant uptake and washout of Sestamibi (Maublant 1988) with the expected decay in isotope of $10 \%$ as shown in Figure 21. During this time, the greater amount of radioactive material present in the normal region provides a concentration gradient for increased delivery of isotope (sestamibi) to the region with critical narrowing and/or vulnerable plaque, adding to the increased delivery in isotope in this region over 
time. This increased delivery of isotope is defined as washin as will be discussed below. The far right hand Section of the diagram shows the effect of decreased retention of sestamibi by ischemic mitochondria (Crane 1993) with a greater than expected $(10 \%$ by technetium- $99 \mathrm{~m}$ isotope decay) reduction in isotope concentration returning from the mitochondria into regions of normal tissue with a greater capacity to retain sestamibi for a greater period of time, or return of the isotope into the vascular space where isotope is delivered for excretion (Sattari 2001) from the body.

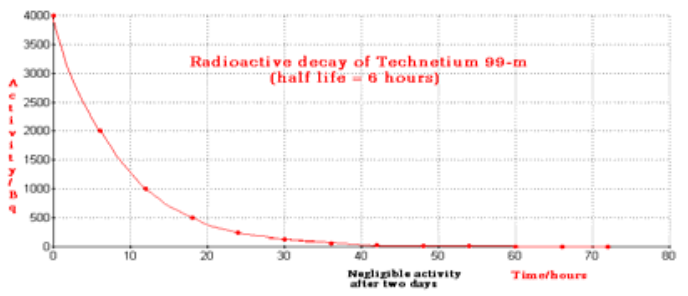

Fig. 21. Decay curve of technetium-99m

Figure 21 shows the decay curve seen for technetium-99m compounds. Over the course of 55 minutes (the time difference between imaging at 5 minutes and 60 minutes), there is a $10 \%$ isotope decay, which will result in the "normal" reduction in isotope seen over a 55minute period of time.

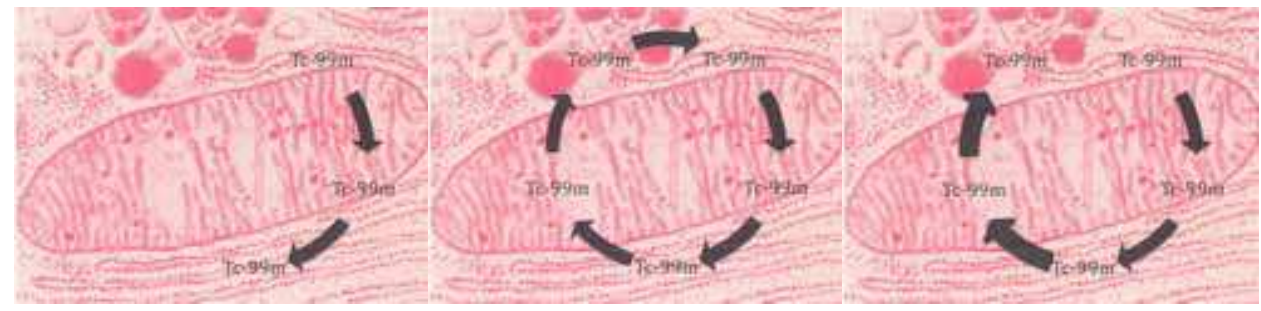

Fig. 22. Changes in mitochondrial movement (redistribution) of sestamibi over time

Figure 22 shows the uptake/release of sestamibi by mitochondria at 60 minutes post-stress. When compared with Figure 18, it is clear that mitochondria (far left) in the critically narrowed/vulnerable plaque regions are now receiving greater concentrations of sestamibi as described in Figure 20. The mitochondria in the middle image shows continual uptake and release of sestamibi given the unimpaired delivery of the isotope and normal mitochondrial calcium levels leaving an unimpeded mitochondrial uptake and release of sestamibi. The far right panel reflects the findings noted in Figure 18, where there is relatively unimpeded delivery of sestamibi to the mitochondria, with increased release due to the effect of ischemia upon mitochondrial calcium concentration and mitochondrial function.

Figure 23 shows the results of sestamibi concentration and measurement of radioactive levels of sestamibi at 60 minutes post-stress. These results show what is described above, viz. and increase in concentration in the region of the vulnerable plaque/critically narrowed coronary artery with a count of 30,470. The region with normal blood flow and normal functioning mitochondria shows a count of 25,472 while the ischemic region without a critical narrowing or vulnerable plaque shows impairment in mitochondrial function as described above with a count of 15,407 despite reasonable delivery of the radioactive isotope sestamibi. This single 
60-minute image minus the actual ROI counts, appears completely normal qualitatively leading one to detect no ischemia when it is used for rest to stress comparisons, which Gorlin (1959) demonstrated could not be used as a method for detecting ischemia.

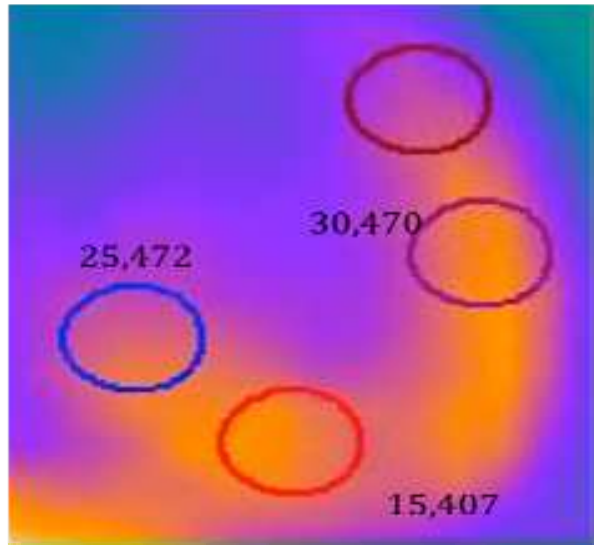

Fig. 23. Measurement of sestamibi redistribution as quantitatively measured, allowing for assessment of both physiologic and anatomic information, which cannot be determined by comparing images using a visual Gestalt

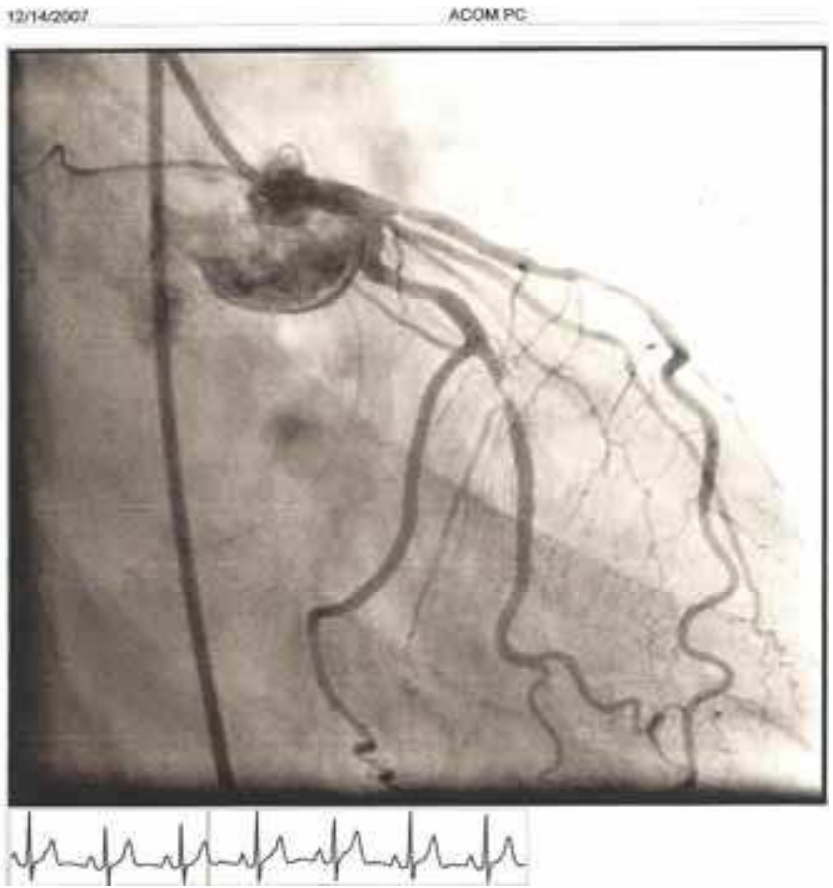

Fig. 24. Anatomic information obtained from coronary angiography which was compared with physiologic information obtained from Sestamibi redistribution 
Figure 24 shows the results of coronary angiography with detection of vulnerable plaques, normal arterial regions and regions of ischemia matching the findings of wash in and washout; but, not matching the results of the single 60-minute image displayed in Figure 23 when using only a qualitative method in stark contrast to a comparison of change seen between the 5-minute and 60-minute images above.

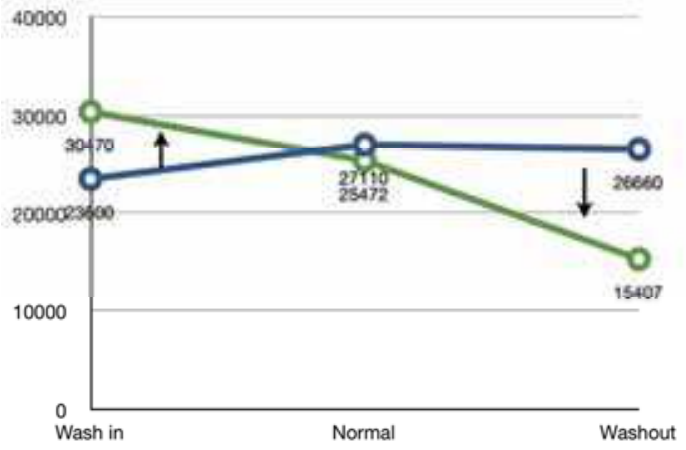

Fig. 25. Comparing quantitative sestamibi information between stress-stress imaging

Figure 25 represents the results of what is seen when the quantitative measurement of ROIs are compared for (a) regions of critically narrowed/vulnerable plaque regions (wash in) where radioactive concentration is seen to increase between the 5 and 60-minute images, (b) when disease is not present (both flow and mitochondrial function are unimpaired) and the change is count activity can be accounted for on the basis of isotope decay (Figure 21). As we have seen, the isotope (sestamibi) is not merely taken up and retained but as demonstrated by both Maublant (1988) and then Crane (1993), the isotope undergoes uptake and release, the rate of which is determined by ischemia and mitochondrial function. Finally, (c) washout is seen as an initial near normal delivery of isotope with impaired retention independent of the delivery of the isotope owing to mitochondrial calcium overload resulting from ischemia.

\section{Stenosis $=0.011 \cdot$ Washout $^{2}$}

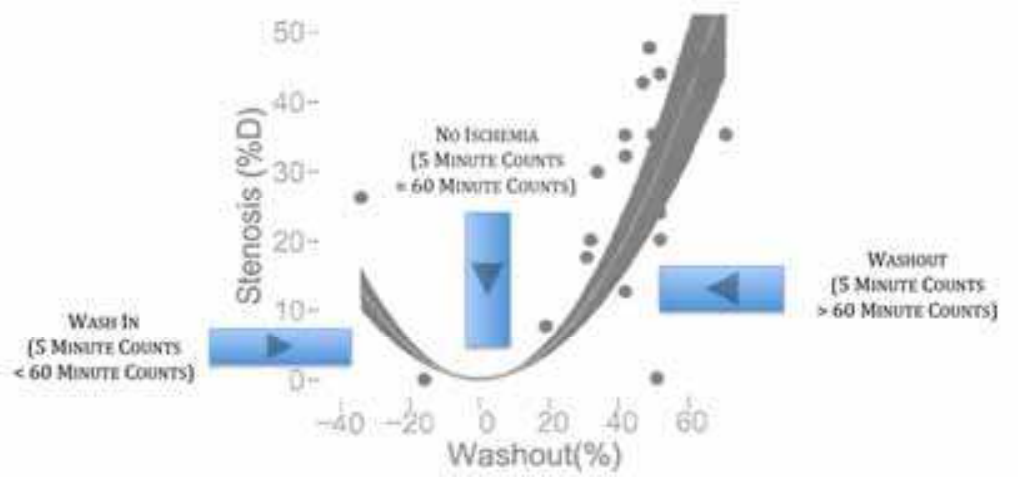

Fig. 26. Single injection, single camera imaging assessment of anatomic AND physiologic information obtainable by quantitative measurement of Sestamibi redistribution 
Figure 26 summarizes all of the information seen both with changes in isotope concentration (washin, normal, washout); but, more importantly compares this with findings seen on coronary angiography, enhancing decision making capabilities from this physiologic change (Fleming 2008b, 2009a) in isotope over time.

Armed with the understanding of how our Nuclear SPECT cameras work and how these isotopes redistribute over time, we can now better understand the process by which we can best use our nuclear cameras to detect ischemic heart disease. It is estimated that in the United States alone, some 10 million nuclear heart scans are performed each yearly. Of these some 80,000 to 140,000 individuals will be told following their (Heart 2011) "reststress" SPECT myocardial perfusion imaging (MPI) studies, that they have no ischemic heart disease whatsoever. Of these 40,000 to 70,000 will go home and die from a heart attack. Evidence indicates that these individuals have "normal" appearing 60-minute poststress studies and are only detected by finding a decreased uptake at 5-minutes post stress, viz. "wash-in." The "rest-stress" approach simply cannot detect these individuals. In the next Section of this chapter, we will look at the evidence proving this and scientifically establish why so many people are being misdiagnosed and what we can do about it.

\section{Fleming Harrington Stress SPECT protocol reduces radioactive dosage and increases ischemia detection. The detection of ischemic heart disease is accomplished by comparing multiple images of the heart following stress. (ischemia equals stress imaging)}

Background: Radioisotope manufacturers, nuclear camera manufacturers, standard guidelines for myocardial perfusion imaging, all assume rapid isotope uptake followed by an essentially static retention mechanism. Contrariwise, the historic first nuclear cardiology study found measurement of nuclear isotope blood flow kinetics to be diagnostic of heart disease. No clinical investigations appear to have followed that classic finding. We assessed the clinical diagnostic utility of time course myocardial perfusion measurements following single injections.

Methods: One hundred twenty patients suspected of having heart disease underwent Sestamibi stress images taken at 5 and at 60 minutes following completion of cardiac stress. Quantitative (FRHWW) redistribution differences between the images were assessed. FHRWW results were compared with angiographic findings.

Results: Parabolic regression of stenosis on redistribution yielded an effect size of R(CI95\%) $=0.72$ to $0.95,\left(P=3.8 \times 10^{-8}\right)$. Fifteen percent of individuals with "normal" appearing 60 minute SPECT images were noted to having coronary artery disease using both redistribution data and coronary angiography requiring stent placement. These individuals had "wash in" of Sestamibi (isotope uptake greater at 60 minutes than 5 minutes) and signaled patients at risk of undergoing an acute cardiac event due to vulnerable plaques or tight lesions placing significant amounts of myocardium at risk of infarction. One of the individuals who did not require cardiac catheterization based upon the FHRWW data, underwent coronary angiography based upon rest-stress results AND had a major adverse event as a result of the subsequent cardiac catheterization. There was no detectable CAD, confirming the FHRWW findings and the need for caution in individuals undergoing invasive procedures.

Conclusions: Dynamic "uptake / release" models appear to be a superior alternative to the common "uptake/retention" models of technetium-99m isotopes used in nuclear myocardial perfusion imaging. This sequential quantitative diagnostic model, reinforces the 
work by Blumgart, enabling more accurate diagnosis of coronary artery disease and providing for intervention in individuals requiring stents or bypass, whom would otherwise be missed on rest-stress imaging protocols while avoiding unnecessary coronary angiography among individuals with "normal" Sestamibi redistribution.

While diagnostic testing and decision making in the treatment of atherosclerotic coronary artery disease (ASCAD/CAD) tends to revolve around evidence of anatomic [cardiac catheterization (Fleming1991b), coronary computed tomography, intravascular ultrasound (Taki 2001, St Goar 1991), et cetera] disease, it has been well established that this inflammatory (Fleming 1999c, 2000c-d) process may smolder for years and that up to $85 \%$ of all myocardial infarctions occur with $<30 \%$ diameter narrowing. It is; therefore, important for us to look for new ways to uncover this smoldering inflammatory disease process when intervention may be more beneficial to the patient and potentially less costly to the patient and society at a time when health care costs are a major issue.

When teenagers learn to buy their first automobile, they learn an important lesson. Not every car that looks good runs well. This same lesson applies to the diagnosis and treatment of ASCAD. The lesson is to determine how well the car runs, or in this case, how well the heart runs. Hence the importance of physiologic testing; i.e. testing to see how well the heart runs. Prior to nuclear imaging of the heart, now called myocardial perfusion (Maddahi 2001, Kern 2006) imaging (MPI), patients ran on a treadmill (exercise stress testing/EST) under the belief that this would precipitate chest pain (angina) and perhaps electrocardiographic and hemodynamic changes which would indicate problems with coronary blood flow. Until recently, it was believed that this was purely the result of narrowing within the lumen of the artery. It has since been demonstrated that angina is the result of regional blood flow (Fleming 2000e, 2003d) differences; hence, the importance of quantifying such areas of the heart by analyzing regions of interest (ROI). These differences in regional blood flow work through mechanisms as of yet not completely understood. The ability to precipitate these differences in regional blood flow can be accomplished via the utilization of MPI and pharmacologic or exercise stress testing. A major limitation with utilizing single 60 minute images for interpreting the presence of ischemia on MPI are attenuation artifacts. Such artifacts include anterior attenuation from breast tissue, diaphragmatic attenuation from abdominal obesity, hypertrophic differences in walls of the heart, bundle branch anomalies, et cetera. These attenuation issues have not been significantly corrected with the addition of attenuation correction protocols. While nuclear tracers (isotopes) have improved over the years, many misconceptions (Beller 1990, Fleming 1991a, Sinusas 1994, Dahlberg 1994, Glover 1995, Hurwitz 1996, Husain 2007) have plagued the field limiting the accuracy of MPI with sensitivities and specificities remaining at $65-90 \%$ in most published investigations (Parameswaran 2006).

In 1926 Blumgart first proposed nuclear imaging techniques for the detection of heart disease. His method clearly proposed serial assessment of isotope count activity to determine cardiac function. Decades passed before Love (1965) discussed the potential future of Nuclear Cardiology and pointed out that rest images (Gorlin 1959) were useless in the detection of coronary artery narrowing. With the advent of technetium-99m- sestamibi, clinicians believed that the isotope was taken up by myocardial tissue within minutes of injection and remained fixed within myocytes. Crane (1993) demonstrated this was not so and demonstrated that retention of sestamibi was dependent upon ischemia and cellular viability. This opened the door to the discovery of changes in isotope count over the course of time and reinforced Blumgart's 1926 paper. During our initial (Fleming 2003e, 2008) efforts to better understand the underlying inflammatory component of CAD via MPI, five 
and sixty minute imaging revealed differences in the qualitative appearances of images seen following pharmacologic stressing of individuals as predicted by Blumgart (1926), Love (1965) and Crane (1993). As noted below, others have noticed these differences as well. Furthermore, comparisons of the initial 5-minute imaging with the 60-minute imaging demonstrated different results from the simple "uptake/retention" rest-stress model. Other investigators have noted similar differences between 5 minute and 60 minute imaging of the heart (Hurwitz 1993, 1998, Saha 1994, Giubbini 1995, Pace 2005) allowing for enhanced detection of congestive heart failure (Hurwitz 1998, Kumita 2002, Sugiura 2006, Matsuo 2007), cardiomyopathies (Meissner 2002, Ikawa 2007), Prinzmetal 's angina (Ono 2002, 2003) and underlying coronary artery disease (Meerdink 1990, Richter 1995, Shin 1995, Takeishi 1996, Takahashi 1996, Fujiwara 1998, Hurwitz 1998, Ayalew 2000, 2002, Liu 2001, Kumita 2002, Tanaka 2006, Fukushima 2007, VanBrocklin 2007) including evidence of wash-in (Meerdink 1990, Richter 1995) indicative of critical lesions not detected by conventional MPI. In the same way that thrombolysis in myocardial infarction (TIMI) flow is used to look for changes (sometimes subtle, sometimes not) in coronary blood flow in the catch lab, multiple imaging following pharmacologic or EST allows us to take a more advanced look at the physiologic function of the heart, unmasking ischemic heart disease missed by rest-stress imaging.

Given these recent investigations into improving the detection of ischemia by quantifying isotope counts on multiple (sequential) images following pharmacologic and exercise stress, we set out to determine (1) are nuclear cameras used in the "clinical" setting today able to detect differences in radioactive isotope counts over a period of time, which would allow these cameras to detect changes in flow and retention of isotope over time (2) what are the optimal imaging times for the detection of ischemia and (3) how do these changes in isotope counts compare with percent diameter stenosis as seen on coronary angiography?

\subsection{Methods}

\subsubsection{Single Photon Emission Computed Tomography (SPECT) cameras}

The Philips Forte Dual Head SPECT camera used general all (GAP) purpose collimators with the heads positioned at 90 degrees per manufacturers specifications. A $15 \%$ window was used with a 64 by 64 matrix. In the first Section of this study, we additionally looked at differences between $64 \times 64$ matrix and $128 \times 128$ matrix. Imaging occurred 5 and 60 minutes post stress with processing of images as described in the literature (Fleming 1991a, 2003e, 2008). Jet stream software was used per manufacturer's instructions to quantitatively measure regions of interest (ROIs).

The Picker Axis Dual Head SPECT camera used a low energy general all (LEGAR-PAR) purpose collimator with parallel hole positioning. The heads were positioned at 102 degrees per manufacturer specifications. Picker camera software was used for ROI measurements.

1. Determining if Nuclear Cameras used in "Clinical" Practice are able to detect changes in isotope count over time.

Establishing technetium-99m decay by radioactive count measurement: Based upon the half-life of technetium-99m, a stable source representing the "uptake/retention" model (viz. a syringe filled with the isotope) has a $10 \%$ decay over 55 minutes with a retention of $\sim 90 \%$ of the radioactive counts over a 55 minute (the difference between 5 and 60 minute images) span of time. In an effort to establish whether "clinically" used cameras are able to measure this physical decay in technetium- $99 \mathrm{~m}$, we studied a $37.37 \mathrm{MBq}$ (10.1 mCi) sample of sestamibi at baseline and again 55 minutes later. As shown in 
Figure 10, the resting isotope count was 1,405,721 while the second image obtained 55 minutes later was 1,251,359 representing $89 \%$ of the original count activity. Independent of the actual amount of activity which is taken up by a region of myocardium, if the "uptake/retention" model was correct, images taken 55 minutes after the original image would yield counts of $90 \%$ of that seen in the first image.

Fleming-Harrington Redistribution Wash-in (FHRWW) Washout (Fleming 2009a-b, 2010a): Using Multiple SPECT Camera Images of the Heart to Determine Changes Between 5 and 60-Minute Images.

2. Optimal Timing to Measure Changes in Radioactive (Sestamibi) Isotope Activity.

Measuring regions of interest (ROIs) to quantify changes in technetium-99m isotopes between 5 and 60-minute images to look for changes in isotope "uptake/retention." We began with the knowledge acquired from the first Section of this study, that we needed to use the $64 \times 64$ matrix to accurately measure changes in radioactive decay. We then proceeded to analyze ROIs at 5-minutes (Figure 27) and at 60-minutes (Figure 28). FHRWW calculations (discussed below) demonstrated that the "uptake/retention" model was clearly wrong as seen by the increase in count activity in the anterior wall (23,600 at 5-minutes and 30,470 at 60 minutes) while the inferior region without ischemia demonstrated a $6 \%$ reduction in count activity.

Establishing the optimal time sequence for image acquisition to measure radioactive counts: Using this particular individual (Figures 27 and 28), during this second phase of the investigation, we additionally compared the basal anterolateral, mid anterolateral, basal anterior, mid anterior, basal inferior, mid inferior basal, inferoseptal and mid inferoseptal ROIs and compared the results with that obtained from coronary angiography. We then measured radioactivity in each of the 8 ROIs over the course of an hour, with the salient findings reported in Figure 29. These results were compared with our previous studies (Fleming 2003e, 2008) which revealed the ideal timing for detection of inflammatory changes and based upon the expected $10 \%$ decay of technetium-99m over a span of 55 minutes, the remainder of our work (FRHWW) focused on ROIs obtained at 5 and 60 minutes post stress. These results confirm that Sestamibi does NOT follow an "uptake/retention" model; but rather an "uptake/release" model consistent with Sestamibi redistribution (FHRWW).

\subsection{Calculating FHRWW}

By comparing 5 and 60 -minute imaging, the expected decay of Tc- $99 \mathrm{~m}$ is $10 \%$. Actual counts from individual ROIs can then be taken and actual redistribution (FHRWW) can be calculated as follows.

FHRWW (Total Heart) =

$=\frac{\text { ROI counts }(\text { total heart }) \text { at } 5 \text { minutes }- \text { ROI counts }(\text { total heart }) \text { at } 60 \text { minutes }}{\text { ROI counts (total heart) at } 5 \text { minutes }} \times 100-10$

Where 100 places the answer in percent minus the $10 \%$ expected decay.

FHRWW for a particular myocardial ROI =

$=\frac{\text { ROI counts (region) at } 5 \text { minutes }- \text { ROI counts (region) at } 60 \text { minutes }}{\text { ROI counts (region) at } 5 \text { minutes }} \times 100-10$ 
This can be re-written as:

Let the 5 minute counts $={ }^{\prime} x$ ', let the 60 minute counts $={ }^{\prime} y$ ', and let $\%$ washout $={ }^{\prime} 100 * w^{\prime} \quad$ (eq 3)

Then $\mathrm{w}=(\mathrm{x}-\mathrm{y}) / \mathrm{x}-0.1=\mathrm{x} / \mathrm{x}-\mathrm{y} / \mathrm{x}-0.1=1.0-\mathrm{y} / \mathrm{x}-0.1=0.9-\mathrm{y} / \mathrm{x}=.9-60$ minute counts $/ 5$ minute counts.

3. Comparing washout/wash-in results with coronary angiographic assessment of percent diameter stenosis.

One hundred twenty patients (ages 25 to 82 years) with suspected coronary artery disease underwent myocardial perfusion imaging after signing informed consent per Institutional protocol following adenosine $(n=30)$, lexiscan $(n=61)$, dobutamine (Fleming 1995, 1999a, Calnon 1997, 1999, Matsunari 2001), n=4 or treadmill (Fleming 1991a, 1999a), n=25 stress. Following standardized protocols already published (Fleming 2009a-b, 2010a) MPI using both the rest-stress and stress-stress (FHRWW) approach were compared with coronary angiography (Fleming 1992a-b).

Statistical Analysis. Quantification of isotope counts was made using the specific nuclear computer software provided by the nuclear camera companies. These quantified counts were recorded with calculations of FHRWW as noted above. Correlation coefficients, confidence intervals and p-value (significance levels) were determined between \%DS and FHRWW using product-moment correlation and least squares regression fitting the hypothesized model of $y=\mathrm{cx}^{2}$. Using the null hypothesis for $n=120$ patients, we estimated odds ratios (ORs) for predicting final diagnosis from nuclear procedures. ORs were determined against coronary angiography results for both the rest/stress images and FHRWW images. Statistical analysis and graphics were developed using R-2.6.0 and GGobi software.

\subsection{Results}

To the best of our knowledge verification of the ability of a nuclear camera used for "clinical" purposes to measure sequential radioactive counts confirming differences in technetium-99m-sestamibi resulting from the physical decay of the isotope has not previously been reported in the literature. The first phase of this study was to confirm that cameras used clinically are able to detect differences in radioactivity accurately, assuming they employ the correct matrix to do so. To prove this, we used $37.37 \mathrm{MBq}$ of technetium$99 \mathrm{~m}$-sestamibi with both a $64 \times 64$ matrix and a $128 \times 128$ matrix. The results of the $64 \times 64$ and $128 \times 128$ matrices are shown in Figure 10. Over a 55 minute period of time, the expected decay of technetium- $99 \mathrm{~m}$ would be $10 \%$, leaving a residual activity of $90 \%$ of the original counts. This represents a true "uptake/retention" model since the radioactive compound is retained within the syringe. Using the $64 \times 64$ matrix, the radioactive counts went from $1,405,721$ to $1,251,359$ representing an $11 \%$ decay with a residual $89 \%$ of the original isotope activity. The $128 \times 128$ matrix reported 3,473,001 at baseline and a count of $2,966,39455$-minutes later for a decay of $14.6 \%$ with a residual of $85.4 \%$ of the original isotope activity. For this reason, the $64 \times 64$ matrix more correctly demonstrated the expected decay of the isotope based upon the physical half-life of Tc- $99 \mathrm{~m}$ and was therefore chosen as the standard for performing these studies.

The second question related to the timing of data collection. Prior studies (Fleming 2003e, 2008) demonstrated the ability to detect inflammatory markers on SPECT images at 5 minutes; but, not at later periods of time. Figure 29 displays the results of radioactivity measured using the clinical camera with a $64 \times 64$ matrix. As shown, the peak detectable 
radioactivity was measured between 5 and 10 minutes post injection for individuals with "no" coronary artery disease or coronary artery disease without "critical" lesions or "vulnerable" plaques. In individuals with "critical" lesions and/or vulnerable plaques, radioactive counts increase from 5 to 60 minutes, viz. wash-in (Fleming 2009a-b, 2010a). Combined with the results of instrumentation (Figure 10), the ability to detect ischemia based upon blood flow and cellular uptake and release of sestamibi, is best made by comparing counts at 5 minutes and 60 minutes.

The final component of our study compared results of rest-stress imaging and FHRWW with angiographic (\%DS) data. There were no differences observed between individuals who were stressed either physically or pharmacologically. Outcomes were not changed by the stressor used, as the comparison between rest/stress and FHRWW determination of redistribution was independent of the type of stress. The use of regional wall motion information was used with both the rest/stress protocol and the FHRWW approach, which made it possible to look for wall motion abnormalities and ejection fraction using either approach. Since these observations are identical, regardless of which method was used to report ischemia, we do not report them here.

We analyzed the results of rest/stress image comparisons and FHRWW redistribution images and compared them with findings obtained from coronary angiography. For the rest/stress images, the OR for detecting ischemia was 4.9 with a CI (95\%) of 2.3 to 10.3 . For FHRWW, the OR was 56.8 with a CI (95\%) of 27.5 to 117.2. When the results of these ORs were compared, the $t$ value was greater than $6.6(\mathrm{P}<.0001)$. We obtained results of \%DS for each of the epicardial arteries and their branches, and plotted these results against the redistribution results for each of the eight ROIs. This could only be done with the redistribution data, which provided quantitative results, which could be compared with the quantitative results obtained angiographically.

Comparison of the rest/stress image results with the angiographic results showed a sestamibi rest/stress imaging sensitivity of $67 \%$. Of the false negative results ( $n=22), 18 \%$ ( $n$ $=4$ ) had critically narrowed arteries or arteries with vulnerable plaques whose 60 -minute images appeared completely normal (Pace 2005, Fleming 2008). However, evaluation of the 5-minute images for redistribution (using FHRWW), revealed both qualitative and quantitative decreased uptake, demonstrating a washin effect. The 60-minute post stress images miss this washin phenomena when looked at independent of the 5-minute images, yielding incorrect results. The standard rest/stress images also yielded a specificity of $88 \%$; of which, only one participant $(0.8 \%)$ who underwent a coronary angiogram had an adverse outcome requiring cardiopulmonary resuscitation (Hurwitz 1993).

FHRWW calculation was made first by comparing the total radioactive counts of the heart at 5 minutes with the total counts of the heart measured at 60 minutes. The eight regions of interest (ROIs) were then compared using both the 5 and 60-minute image information. Analysis of eight ROIs appears to provide the optimal analysis of specific coronary artery beds (left anterior descending, circumflex and right). These regions include the basal anterior, mid anterior, basal anterolateral, mid anterolateral, basal inferior/posterior, mid inferior, basal inferoseptal and mid inferoseptal. Examples of some of these regions are shown in Figures 27, 28, 30A-C and 31A-D. These multiple examples show how, independent of different color scales and cameras used to qualitatively estimate ischemia; quantitative measurements can be made independently and without interference from hepatic and other tissue uptake. Washout (counts greater at 5 minutes than 60 minutes, excluding the expected isotope decay) and washin (noted counts greater at 60 minutes than 
5 minutes) were plotted against the percent diameter stenosis (\%DS) determined on coronary angiography. Washin was seen in $15 \%$ of the individuals studied and was associated with "normal" appearing 60-minute images. When the results of washout/washin were compared with \%DS, a parabolic relationship (figure 6) was demonstrated with $\mathrm{F}=70.4, \mathrm{df}(1,21),\left(\mathrm{P}=3.8 \times 10^{-8}\right), \mathrm{R}(\mathrm{CI} 95 \%)=0.72$ to 0.95 .

\subsection{Discussion}

The advent of nuclear cardiology by Blumgart beginning in 1925 yielded a new era of medicine with the use of radioactive materials to perform physiologic testing which requires sequential measurements as described by Blumgart to be performed correctly. Unfortunately, decades elapsed (Love 1965) before such nuclear materials were made readily available for "clinical" use. The first agent being Thallium-201 (Tl-201) was plagued with limitations including a long half-life (72 hours) resulting in the need to use relatively low doses (6.8-11.1 MBq) of the isotope. Given the limitations (Fleming 1992a, 1995) of Compton scatter, tissue attenuation and the emission of mercury $x$-rays from the isotope, cameras could not acquire adequate counts for imaging until 60 minutes had elapsed. Given the passage of time, much of what Blumgart discussed had been lost to history (Love 1965). With the advent of Molybdenum-99, Technetium-99m generators, the ability to perform clinical studies using technetium tagged agents (e.g. teboroxime, sestamibi, myoview, et cetera) with shorter half-lifes (6 hours) allowing greater doses (up to $1110 \mathrm{MBq}$ ) and less Compton scatter and tissue attenuation issues yielded what "appeared to be cleaner images". These images were still plagued with attenuation issues and were reported to be "taken up and retained" within minutes of intravenous injections. Despite the report by DuPont (Crane 1993) and multiple other research and clinical (Everett 1977, Fleming 1992b, 2003e, Aodah 2007, Fallahi 2008) studies discussed above and below, which have clearly established that these isotopes redistribute and do not "stick", most clinicians have continued to ignore Blumgart's teaching of performing sequential evaluations under same state conditions and have subsequently limited their investigation of coronary artery disease to single post-stress imaging despite the teaching of nuclear imaging which notes that "a better indicator of organ function is the change in the activity in a particular organ with time." Newer clinical observations and publications (Sheikine 2010) have since been made confirming our earlier observations from 2000-2001.

Inconsistencies in analysis of cardiac imaging have led some researchers (Fleming 2002b, Toriyama 2005, Oregon 2008) to recommend that these clinical studies be standardized quantitatively; something this study has accomplished. In medical school we were taught that there were two ways to understand the results of a test. The first is to compare it against the population (e.g. one obtains an electrocardiogram to look for evidence of ischemia or infarction), the second is to compare it with the results of that test which the patient had previously (e.g. poor R-wave progression may actually represent loss of Rwaves and evidence of a prior anteroseptal injury/infarction which can be made more confidently in the presence of a change in the electrocardiogram for that patient, opposed to the confidence one has in making such a diagnosis when this is the first electrocardiogram). The same problem applies with looking at a region of the heart on a single 60 minute image post stress. Issues of tissue attenuation including (1) breast artifacts in the anterior wall, (2) diaphragmatic attenuation inferiorly, (3) differences in wall thickness (asymmetric septal hypertrophy) and (4) conduction disturbances influencing timing of systole (left bundle branch morphology) are examples of errors made when one region of myocardium is 
compared with another region (e.g. a single 60 minute image) instead of comparing a region with itself (5 and 60 minute images) and looking for change. Unless there is a change in breast tissue or abdominal obesity between the 5 and 60 minute image, errors resulting from soft tissue attenuation of photon activity are eliminated. The same applies for changes in wall thickness and conduction issues; unless there is a change between the 5 and 60 minute image, the region is being compared with itself and such artifacts are eliminated.

Despite an absence of research to support the ability of "clinical" nuclear cameras to accurately detect changes in radioactive counts which match the expected decay of the nuclear isotope in question clinicians have used these cameras to assess the presence or absence of disease in patients. As Archie Cochrane and history has taught us it is truly dangerous to assume that the way we have been doing something is the correct way to do it in the absence of data to confirm we are right. The first Section of this study demonstrated that the cameras we used in this study were able to accurately detect the decay of technetium-99m-sestamibi over a 10\% decay. This was best appreciated using the $64 \times 64$ matrix. Even though manufactures support the use of $128 \times 128$ matrix as giving greater detail, the use of the $128 \times 128$ matrix reported a greater decay in the isotope than is physically possible suggesting that information is lost; perhaps as a result of "modulation transfer function" (a concept well known to photographers and imaging engineers).

During the second and third Section of our study we looked at the timing sequence, which would yield the most valuable information, not only regarding detecting changes in radioactivity with cardiac tissue, but the ability to detect markers of inflammation (Fleming 1999c, 2000c, 2003e, 2008, Kern 2006). Our previous findings clearly demonstrated the importance of looking for markers of inflammation when detectable, i.e. at 5 minutes post stress. During this study and our most recent work (Fleming 2009a-b, 2010a) we clearly demonstrated that in individuals who had ischemia with non-critical narrowing of arteries, that maximum radioactivity was present during the first 5 to 10 minutes. The inability to maintain the Sestamibi in regions of ischemia has previously been termed "washout" and represents an inability to retain the isotope within the mitochondria in the region. This clearly confirms the work of Crane (1993), who demonstrated that sestamibi redistributes AND is not retained in regions of "ischemia." Our studies also proved this to be the case, independent of which technetium-99m isotope (Fleming 2009a) was used or which form of stressor (Fleming 2009a-b, 2010a) was used. Our current study clearly demonstrates this to be true in the "clinical" setting and disproves the "uptake/retention" theory in favor of an continual FHRWW "uptake/release" model which would explain not only the loss of isotope to an ischemic myocardial region over time; but, also accounts for "wash-in." "Wash-in" was noted in 15\% of the cases where regions of myocardium were supplied by "critically" narrowed arteries and arteries whose "vulnerable plaques" were ready to rupture. In these individuals the combination of severely disturbed flow through critically narrowed and/or unstable coronary lumen passages and relatively large regions of ischemic myocardium with impaired ability to accumulate sestamibi, results in a delay in initial isotope counts. From this perspective, uptake is a function of isotope availability (myocardial blood flow) and cellular health; retention is transient with release of the isotope back into the blood stream or redistribution to other cells, a function of cellular health and re-uptake subject to a cellular refractory period. As a result the delay in isotope delivery is not noted if stress imaging is limited to 60 minute imaging. These individuals, despite "normal" appearing 60 minute images, were at risk of experiencing an acute coronary event with loss of substantial amounts of myocardial tissue. 


\subsection{Conclusion}

The standard "uptake/retention" model was observed only in subjects who demonstrated no evidence of coronary ischemia or infarction. In reality, such an "uptake/retention" model is simply an illusion. It is the rapid "uptake/release", "uptake/release", "uptake/release" in non-ischemic, non-infarcted myocardial tissue that makes it appear that the isotope is taken up and retained. In other words, if the amount being released and the amount being taken up are equal, one will not detect a change in isotope count over time, minus the slight decrease occurring from isotope decay. Such individuals would appear to have no change in isotope concentration despite continual turnover of isotope. "Uptake/release" was demonstrated via washout in individuals with coronary ischemia and a delayed "uptake/release" in individuals with washin due to critically narrowed and unstable plaqued regions supplying significant amounts of myocardium. Wash-in and washout are terms used to convey different types of CHANGE in isotope concentrations which occur over time at different rates to indicate the type of lesions present in the individual being studied. The results of these changes can be compared with changes found on cardiac catheterization as shown in Figure 32. As we continue to expand the number of individuals being studied with this method, it is clear that specific regional information can be determined from the parabolic equations used here and graphically displayed in Figure 32. The utilization of this knowledge will allow us to better determine which patients will benefit most from coronary angiography and has now been confirmed "qualitatively" by investigators at UCLA and Harvard Universities (Sheikine 2010). With the advent of nuclear cameras capable of faster image acquisition, we will undoubtedly be able to compare washout of the more rapidly redistributing technetium-99m compounds such as Teboroxime (Fleming 1990a-c, 1991a, 1992b, Goldstein 1991). Given the failure of rest-stress imaging to deliver on the promise of detecting ischemia and tissue viability due to their failure to image the heart as explained by Blumgart (1926) and Gorlin (1959), it is clear that FHRWW represents a superior method as we have already seen above and will see below.

We expect that the FHRWW protocol will replace other outdated and incorrectly proposed protocols due to its accuracy, simplicity, and reduction in amount of radioactive material used for imaging allowing for (1) a further reduction in the amount of radioactive material required for ischemic imaging, (2) faster imaging times (3) with FHRWW the ability to calculate \%DS directly from nuclear imaging and (4) when coupled with previously published flow reserve equations (Figure 14), the ability to calculate stenosis (Fleming 1994) flow reserve directly from FHRWW. The Fleming Harrington Redistribution Wash-in Washout quantification of technetium-99m isotopes will allow for the first time (Fleming 2010c), the acquisition of both Anatomic and Physiologic data from a single radioisotope injection, single camera imaging system, including Positron Emission (PET) Tomography (Fleming 1999a), Magnetic Resonance Imaging, or Computed Tomography (CT).

As mentioned in the first Section of this chapter, the desire to know if the patient has undergone myocardial damage or injury (infarction, stunning or hibernation) has long been a question of interest. While, today it may appear to be much less of a concern given the increased availability to measure cardiac enzymes and look for evidence of regional wall motion abnormalities, either by echocardiography, nuclear, magnetic resonance or other imaging modalities, the detection of wall motion abnormalities and elevated cardiac enzymes does not tell us if the tissue damage is full thickness (Figure 14) and permanent, or partial thickness and recoverable. This is the true value of "resting" nuclear imaging. However, just like ischemic imaging, the detection of tissue damage, requires multiple same state condition (viz. rest-rest) imaging, which we will discuss in the next and final Section of this chapter. 


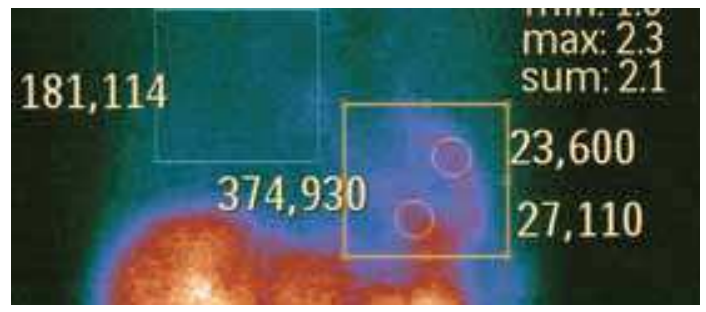

Fig. 27. SPECT image acquired 5 minutes post stress with regions of interest including the total heart, anterior and inferior myocardial regions

Static image acquired at 5 minutes with regions of interest (ROI) showing total heart count of 374,930 . The anterior ROI representing the basal anterior region of the perfused by the left anterior descending artery shows 23,600 counts while the inferior ROI shows 27,110 counts.

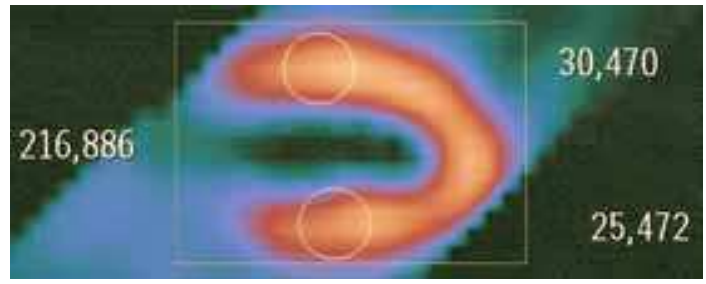

Fig. 28. SPECT image acquired 60 minutes post stress with regions of interest including the total heart, anterior and inferior myocardial regions

Reconstruction of the same myocardial regions noted in figure 2, this time taken from the dynamic image result obtained at 60 minutes post stress, showing a total heart count of 216,886 . The anterior ROI shows an increase in count activity (wash-in) of 30,470 compared with the activity of 23,600 (Figure 2) obtained at 5 minutes. The inferior ROI shows a count of 25,472 compared with the count activity of 27,110 (Figure 2) obtained 5 minutes post stress.

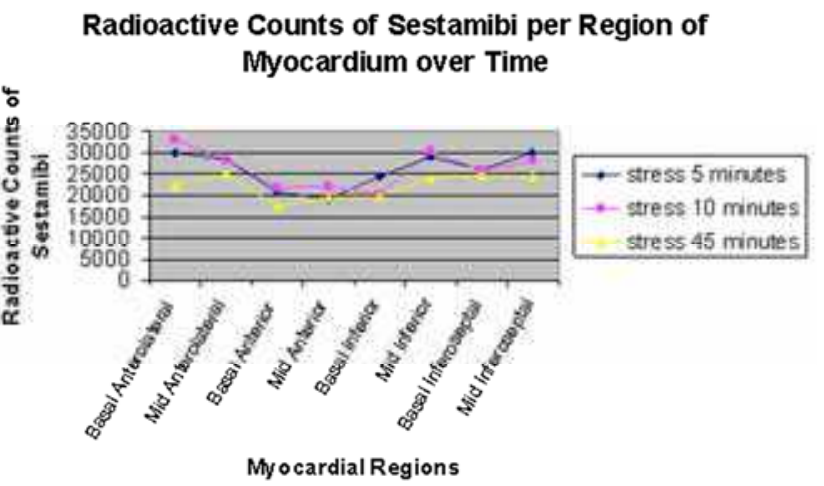

Fig. 29. Determination of Optimal Timing of Image Acquisition

The radioactive counts acquired in eight different myocardial regions vary with time and disease. This graphic demonstrates changes between 5, 10 and 45 minutes post stress. This 
graph demonstrates that the "uptake/retention" model of technetium-99m-sestamibi is incorrect and that "uptake/release" of technetium-99m-sestamibi occurs throughout the first 45 minutes following stress.

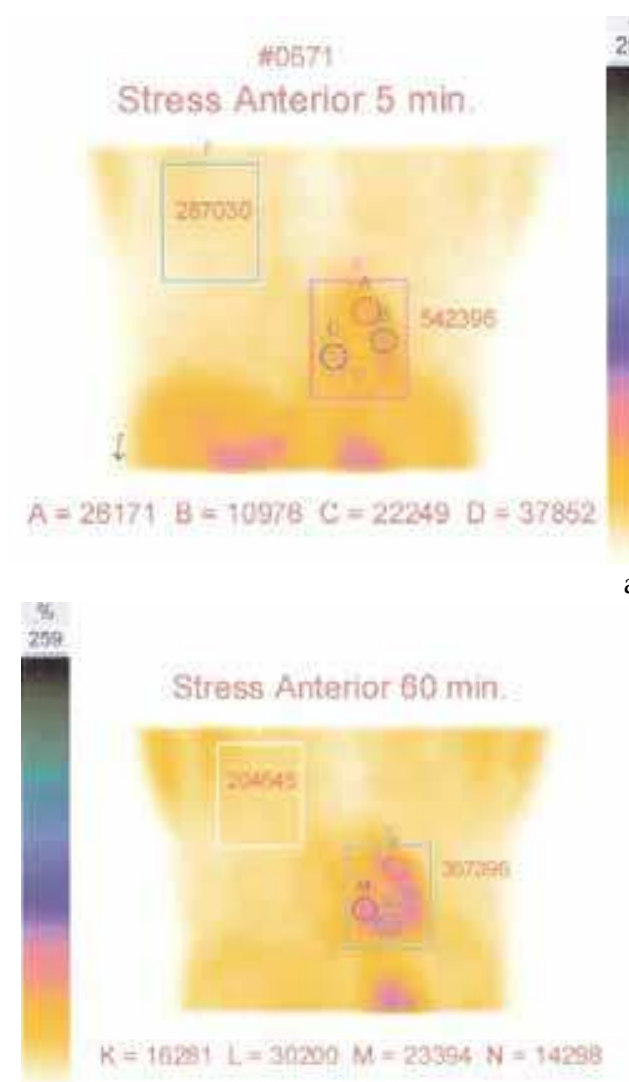

b)

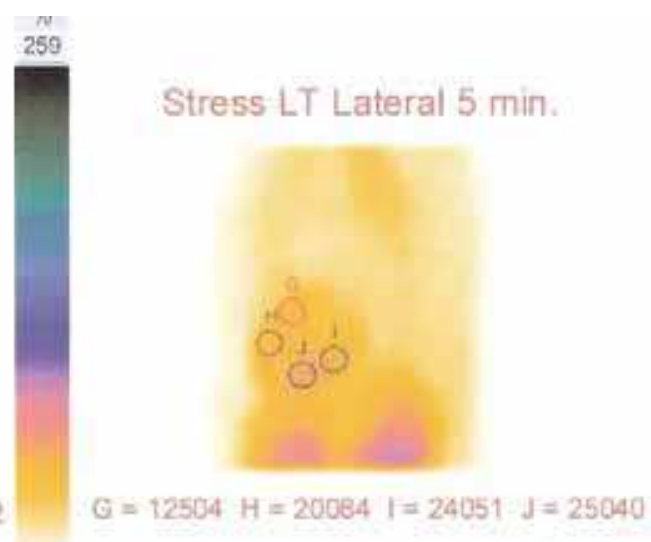

a)

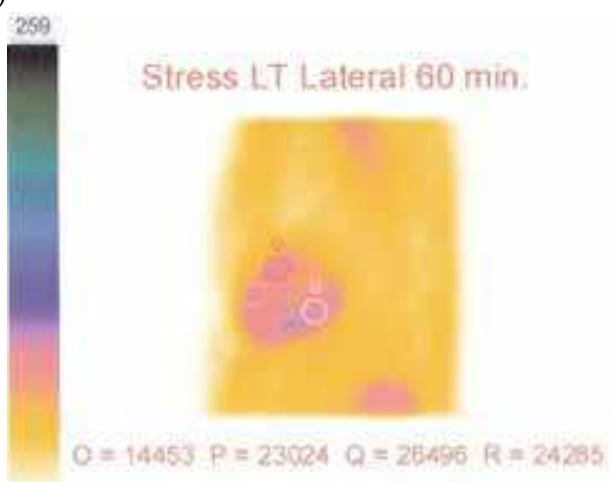

c)

Fig. 30a-c. Radioactive Counts Acquired at 5 and 60 Minutes Post Stress

Anterior images reveal ROI counts at 5 and 60 minutes in the basal anterolateral (A, K), mid anterolateral $(B, L)$, mid inferoseptal $(D, N)$ and basal inferoseptal $(C, M)$ respectively. The counts are shown below the images. The lateral images reveal ROI counts at 5 and 60 minutes in the basal anterior $(\mathrm{G}, \mathrm{O})$, mid anterior $(\mathrm{H}, \mathrm{P})$, mid inferior $(\mathrm{J}, \mathrm{R})$ and basal inferoposterior $(\mathrm{I}, \mathrm{Q})$ regions respectively. The total heart and lung counts are not shown or discussed in this paper; however, their ROIs are represented by square boxes of equal size in the anterior images.

Anterior images reveal ROI counts at 5 and 60 minutes in the basal anterolateral $(1,1)$, mid anterolateral $(2,2)$, mid inferoseptal $(4,4)$ and basal inferoseptal $(3,3)$ respectively. The counts are shown below the images. The lateral images reveal ROI counts at 5 and 60 minutes in the basal anterior $(1,1)$, mid anterior $(2,2)$, mid inferior $(4,4)$ and basal inferoposterior $(3,3)$ regions respectively. The total heart and lung counts are not shown or discussed in this paper; however, their ROIs are represented by square boxes of equal size in the anterior images. 


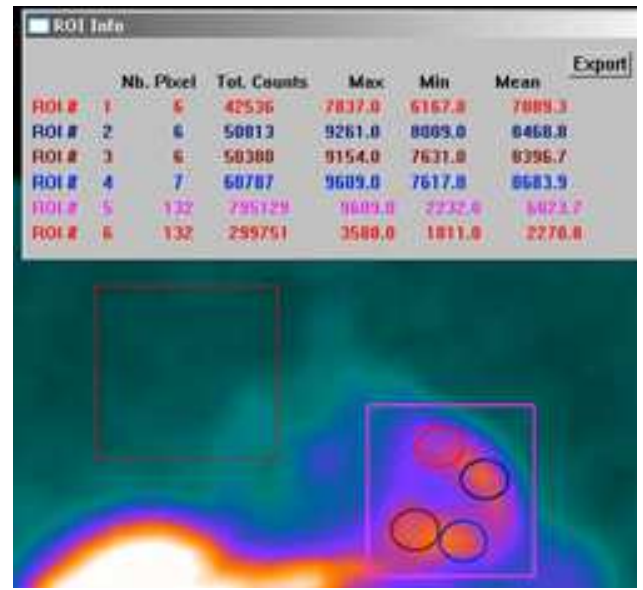

a)

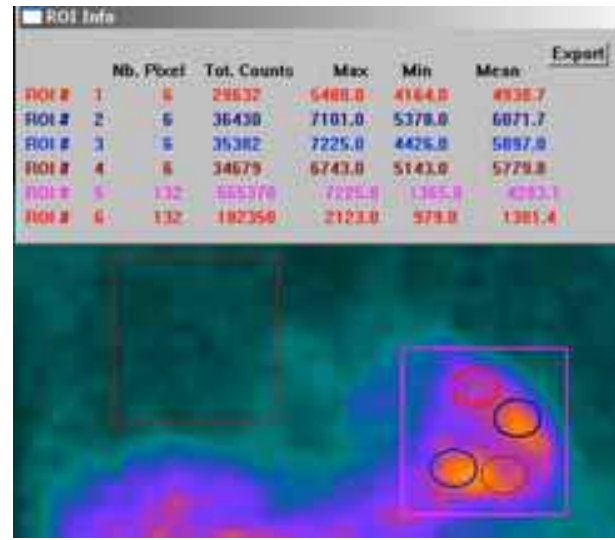

c)

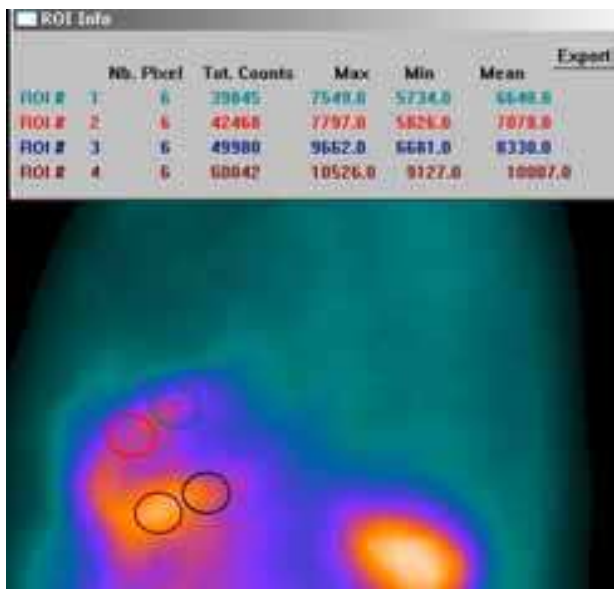

b)

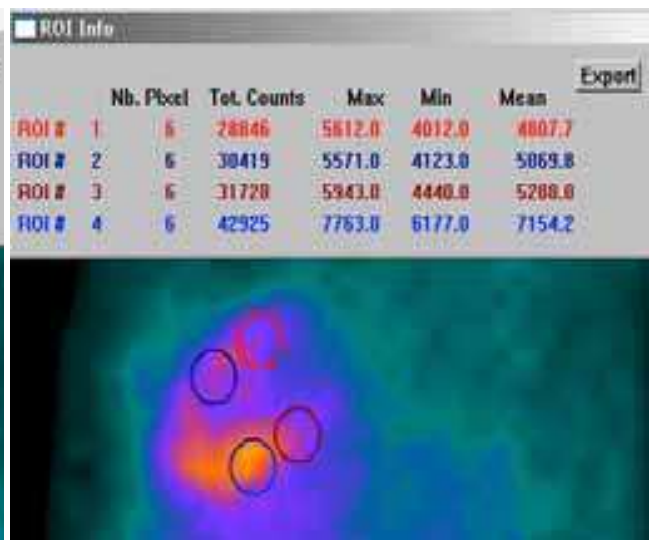

d)

Fig. 31. Radioactive Counts acquired at 5 and 60 Minutes Post Stress

\section{Stenosis $=0.011 \cdot$ Washout $^{2}$}

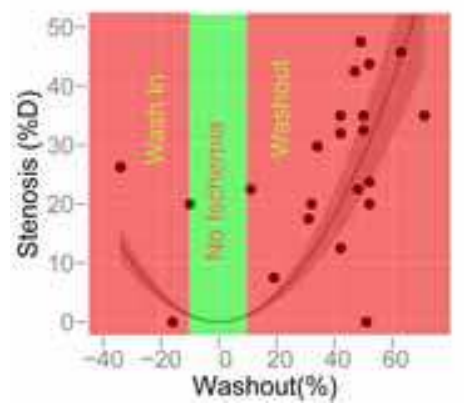

Fig. 32. Parabolic Regression of Washout on Percent Diameter Stenosis 
Percent diameter stenosis noted on coronary angiography was plotted against the results of washout. Stenosis $=0.011 \times$ Washout $^{2}, \mathrm{~F}=70.4, \mathrm{df}(1,21), \quad\left(\mathrm{P}=3.8 \times 10^{-8}\right), \mathrm{R}(\mathrm{CI} 195 \%)=0.72$ to 0.95, Graphic bands show the standard error of the fit. The region of "No Ischemia" is marked in green. "Wash in" is displayed in red to the left of the "No Ischemia" region, while "Washout" is to the right of the "No Ischemia" zone.

\section{FHRWW rest-rest SPECT viability imaging - cardiac viability measured using resting FHRWW redistribution of sestamibi: the scientific evidence proves "sestamibi redistributes and is not mitochondrial superglue"}

Abstract: Sequential imaging of the heart began in 1926 with the emphasis by Blumgart ${ }^{1}$ on detecting changes in isotope over time. In recent years this change in isotope has been defined as "washout/wash in" and has been used to better define ischemia. Our investigations of sestamibi have demonstrated that not only can multiple post-stress images be used to more accurately define ischemia as defined below; but also, multiple resting images can be used to differentiate between viable and infracted myocardium.

The introduction of Nuclear Cardiology in 1926 by Blumgart (1926) established a protocol of sequential imaging/counts to differentiate people with heart disease from those without. In 1959 Gorlin demonstrated the importance of differentiating between resting studies, which provided information regarding tissue damage, from stress studies, which provided important information relevant to ischemia. With the introduction of technetium-99m compounds in the late 1980's, clinicians erroneously assumed that sestamibi was taken up by cardiac tissue within minutes and retained. Crane (1993) proved that the uptake of sestamibi by myocytes was dependent upon mitochondrial calcium, which in turn was dependent upon ischemia. This was confirmed clinically by Hurwitz (1998) and many (Ono 2002, 2003, Fleming 2008b, 2009) others. Our investigations have included initial observations regarding cellular viability. We present a case report of the promise of sestamibi in distinguishing between viable and infracted myocardium.

Case Report. Mr. JW is a 56 year-old Caucasian male who presented to the Emergency Department with chest pressure. His risk factors for heart disease included tobacco use, hyperlipidemia and borderline glucose intolerance. No electrocardiographic changes (Figure 33) were noted. Over the course of the next 24-36 hours both his Troponin and CK-MB levels were noted to be elevated and he was taken to the coronary angiography suite where a $100 \%$ occluded first obtuse marginal was stented. Also noted were a $20 \%$ mid-left anterior descending (LAD) and 50\% distal LAD lesion, which were not treated.

\subsection{Redistribution/washout information}

The patient was brought to the nuclear laboratory 3 days after undergoing coronary angiography to evaluate the extent of myocardial damage. He was given an injection of 370 MBq (10 mCi) of sestamibi and had static images (FH Washout) taken 5-minutes and 60minutes later (Fleming 2008b, 2009). Dynamic images were subsequently taken looking for wall motion abnormalities. The patient then underwent adenosine sestamibi (1110 MBq) imaging following the same protocol as that used for the resting images. The results of the counts obtained from the resting images are shown in Figure 34. The dynamic resting study demonstrated no regional wall motion abnormality and a near absence of isotope activity in the lateral wall. The dynamic stress study showed lateral wall hypokinesis. 
In previous years, technetium pyrophospate was used and later abandoned to define the presence or absence of myocardial infarction (MI) when cardiac enzymes were obtained too late to determine non-q-wave MIs. Of great interest is the ability to determine if an area which appears infracted (Figure 35) on resting studies represents potentially viable tissue, which would benefit from revascularization. Clearly, sestamibi does not simply "stick" to myocardial cells; but, as emphasized by $\mathrm{Crane}^{3}$ is dependent upon mitochondrial calcium, which is subsequently influenced by ischemia. Once cellular death has occurred, mitochondrial function ceases and mitochondrial calcium "overload" results.

In this patient the final 60 minute counts equaled that of the remaining "clearly viable" myocardium and as such is "viable" despite the appearance on the 60-minute resting dynamic study from which only infarction can be inferred. The 60-minute dynamic rest study revealed no regional wall motion abnormality while the dynamic stress study revealed "hypokinesis", not "akinesis". The initial 5-minute static resting counts demonstrated that either injury (viable) or infarction had occurred in the anterolateral region. It is the comparison of the two sets of resting image "counts" which allows the distinction between viable and infarcted tissue. Had the anterolateral region been infarcted, then the myocytes would not have been able to concentrate any sestamibi and both 5 and 60 minute counts would be lower than the remaining viable myocardium. These findings support the speculation by Crane in 1993, that "99m-sestamibi should not be retained in necrotic or irreversibly ischemic myocardium" and that by comparing results at 5 and 60 minutes, we can distinguish between viable and non-viable myocardium.

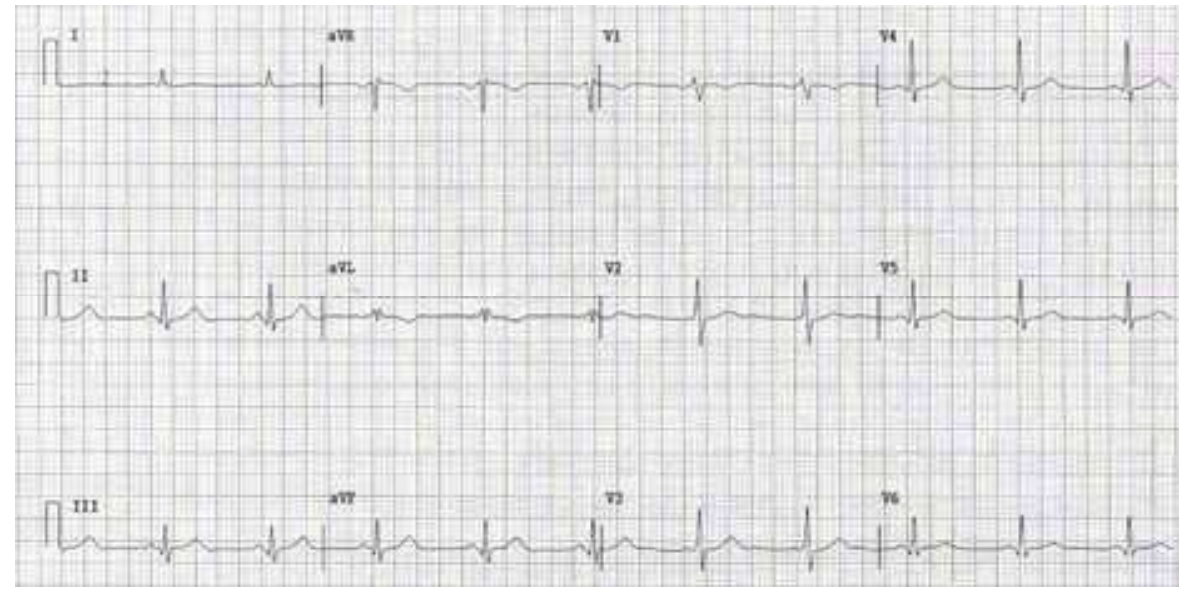

Fig. 33. Electrocardiogram

The patients resting electrocardiogram showed no evidence q-wave or ST changes.

The radioactive counts for each of 8 regions of myocardium are shown. The count activity present at 5 minutes is shown in blue and is greater than that seen for the count activity at 60 minutes. By 60 minutes, most of the myocardium had the same count activity despite the appearance present in figure 3. Note that the count activity in the anterolateral region is much lower initially than the count activity present in the remaining myocardium indicating that a non-q-wave myocardial "damage" has occurred in the anterolateral region. However, the presence of sestamibi activity at 5-minutes indicates viability anterolaterally. 


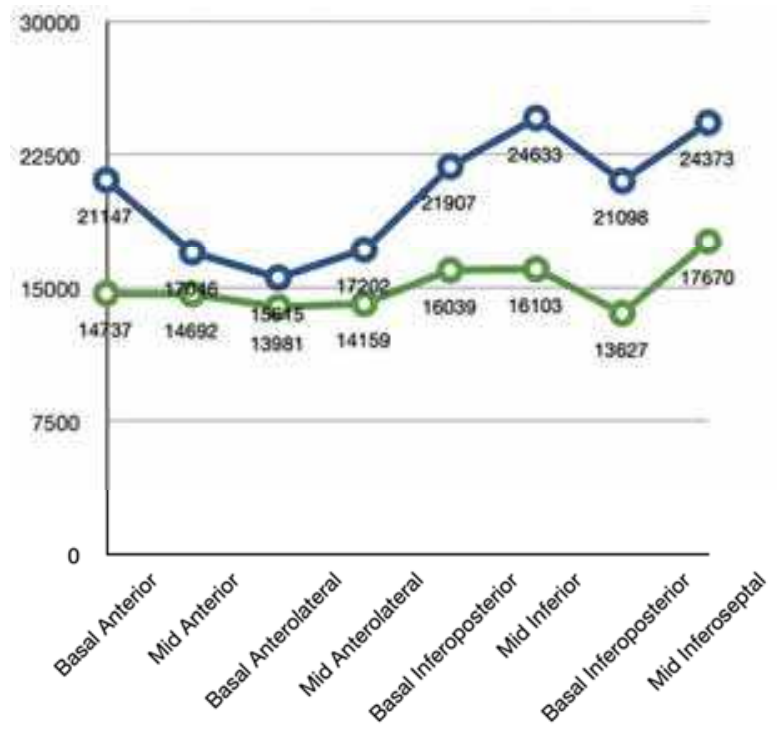

Fig. 34. 5 and 60-Minute Rest Image Counts

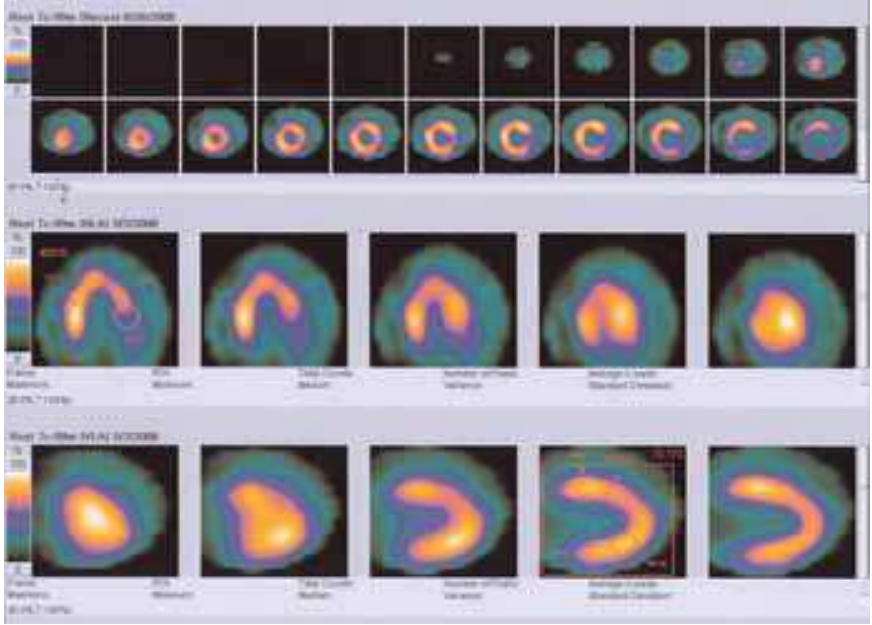

Fig. 35. Results of the dynamic 60-minute rest image

The resting image obtained 60 minutes post injection of isotope shows a significant reduction in isotope in the anterolateral wall despite similar count activity as shown in figure 2. The electrocardiogram in figure 1 shows no evidence of q-wave development or ST changes.

\section{Conclusion}

As this chapter has emphasized, the development of Nuclear Cardiology by Blumgart in 1926 saw a bright future allowing clinicians and scientists the opportunity to look within the 
body by detecting the emission of radioactive decay from tissue following its injection, distribution and redistribution. The radioactive compounds are not static and their movement (redistribution) allows us the unique opportunity to understand where there are and how they change position with time. This movement is a reflection of delivery (blood) and uptake (cellular and mitochondrial) and redistribution of the isotope over time. Just as the solutes within our cells and interstitial space are not static, so it is with the radioactive isotopes we inject. The movement of these radioactive isotopes over time allows us to understand both the Anatomy and the Physiology of the interior of our bodies. More importantly, understanding when to image and for what purpose is key to understanding what processes we are actually measuring (either qualitatively or quantitatively). In the case of ischemia, these comparisons must be made between "stress" images, while for tissue viability, these comparisons must be made under "resting" conditions. While Blumgart and Gorlin were clear as to these points, much of the energy expended in Nuclear Imaging of the Heart has failed to address these errors. As such, coronary arteriography has remained the "Gold" standard for the anatomic detection of heart disease. As our recent understanding has improved, so has our detection of disease and our ability to reduce the amount of radiation our patients receive and that which we receive secondarily. Seventy-five years after Blumgarts original work, we have peered through the uncertainty of our equipment and the limitations of our old methods. Today, we can truly detect heart disease and utilize it to improve the care provided to our patients and be confident of our diagnoses and treatment. We have replaced the "Gold" standard of coronary angiography which can only provide anatomic information of coronary (Figure 36) lumen disease (Fleming 2000f, 2001) with a "Platinum" standard providing both anatomic and physiologic information required for the better detection and treatment (Figure 37) of coronary artery disease.

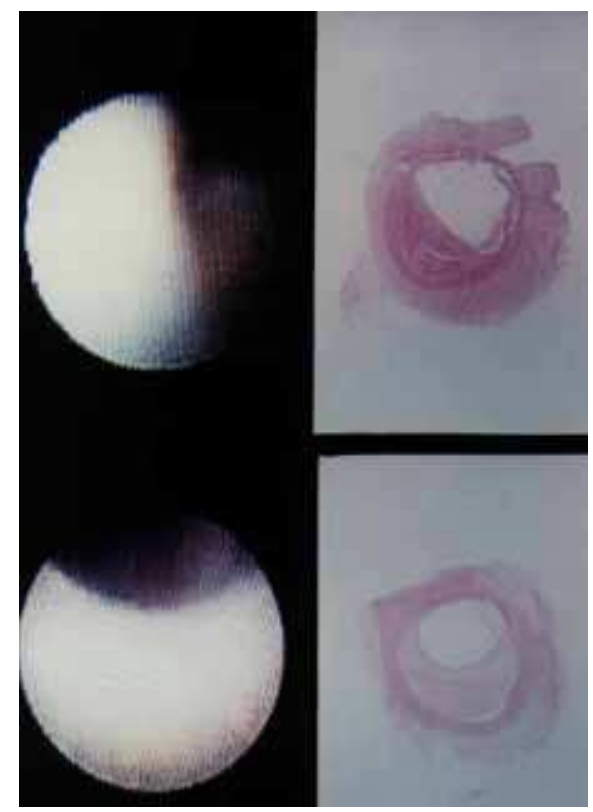

Fig. 36. Coronary angiographic detection of disease 
Coronary angioscopy with tissue specimens show immediately to the right of each following death of the individual. The upper panels show an individual with plaque intruding into the coronary lumen. The bottom panels represent a patient without visible lumen disease. As evidenced by the pathology specimen, the inflammatory plaque laid beneath the endothelial layer and was not detectable by visual inspection of the coronary lumen either by angioscopy or conventional cardiac catheterization and contrast injection. Nonetheless, this inflammatory plaque (Fleming 1999c-d, 2000g, 2002f, 2003d-f, 2008a, $2009 \mathrm{~b}$ ) is physiologically responsible for angina and plaque rupture leading to death. Such individuals may be easily missed by the angiogram and stress-rest approaches; but, can be unmasked by FHRWW stress-stress imaging (Fleming 2009b) as demonstrated in this chapter.

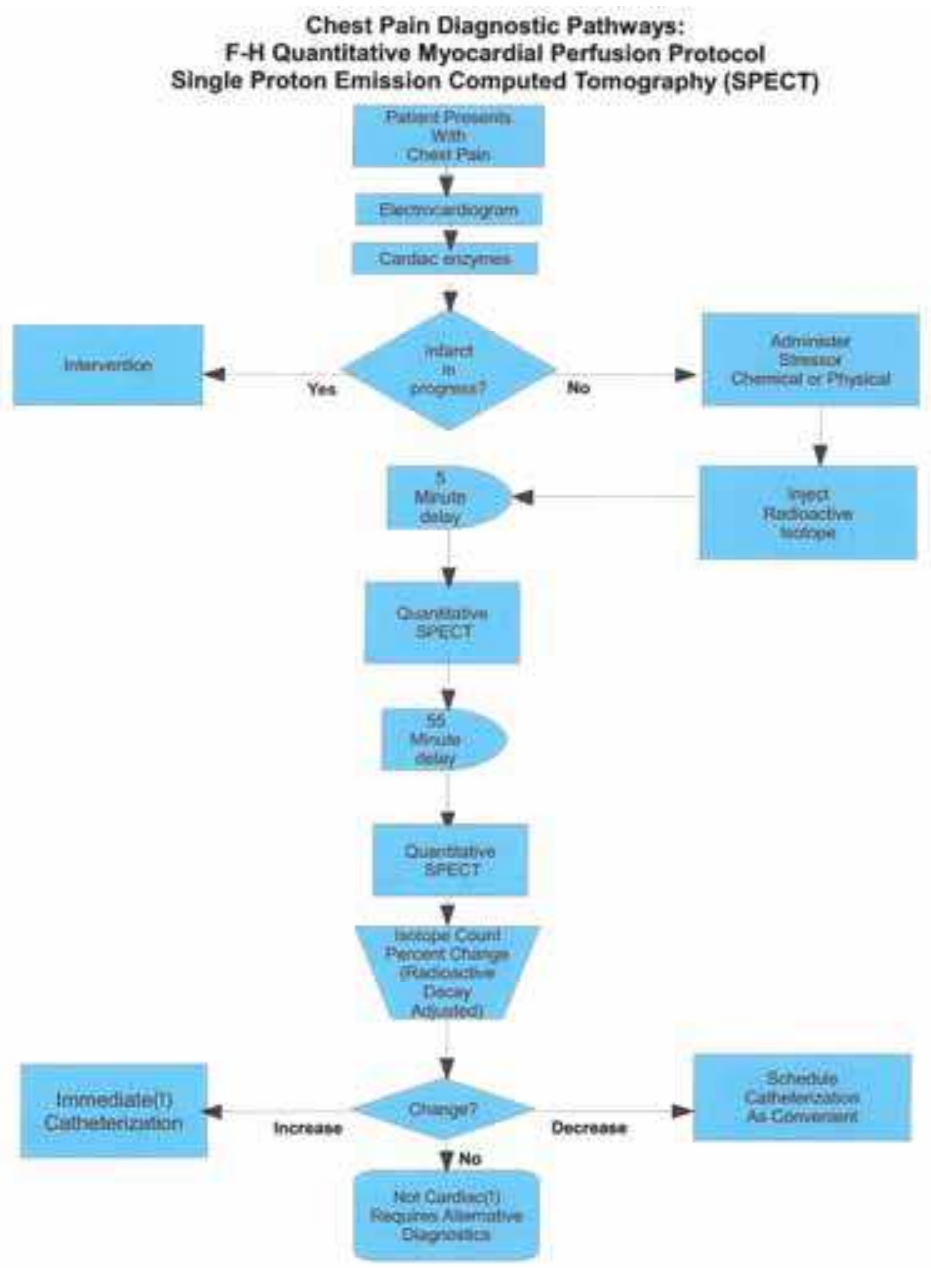

Fig. 37. Chest pain algorithm ${ }^{11}$ using FHRWW 
Efforts to improve acute chest pain analysis employing FHRWW can be used to distinguish between the most critically ill individuals who will have critically narrowed arteries or arteries with vulnerable inflammatory plaques by virtue of wash-in findings defined by reduced isotope at 5 minute post pharmacologic stress imaging and normal appearing isotope concentrations at 60-minutes post-stress from those without such critical disease.

\section{References}

Ahmad M, Logan KW, Martin RH. (1979) Doughnut pattern of technetium-99m pyrophosphate myocardial uptake in patients with acute myocardial infarction: A sign of poor long-term prognosis. Am JCardiol 44:13-17.

Anger H. (1957) A new instrument for mapping gamma-ray emitters. Biology and Medicine Quarterly Report UCRL, 3653: 38.

Aodah B. (2007) "Nuclear Medicine and Gamma Camera" Instructional Course from the King Saud University College of Applied Medical Science, Biomedical Technology DeSectionment. 2007.

Ayalew A, et al. (2000) A comparison of the overall first-pass kinetics of thallium-201 and technetium-99m MIBI in normoxic and low-flow ischaemic myocardium. Eur $J$ Nucl Med 27:1632-40.

Ayalew A, et al. (2002) $201 \mathrm{Tl}$ and 99m Tc-MIBI retention in an isolated heart model of lowflow ischemia and stunning: Evidence of negligible impact of myocyte metabolism on tracer kinetics. JNucl Med 43:566-74.

Beller GA, Sinusas AJ. (1990) Experimental studies of the physiologic properties of technetium-99m isonitriles. Am JCardiol 16:5E-8E.

Blumgart HL, Yens OC. (1926) Studies on the velocity of blood flow: I. The method utilized. JClin Investigation 4:1-13.

Calnon DA, et al. (1997) Effects of dobutamine stress on myocardial blood flow, 99m-Tc sestamibi uptake, and systolic wall thickening in the presence of coronary artery stenoses: implications for dobutamine stress testing. Circulation 96:2353-60.

Calnon DA, et al. (1999) Myocardial uptake of 99mTc-N-NOET and $201 \mathrm{Tl}$ during dobutamine infusion. Circulation 100:1653-9.

Crane P, et al. (1993) Effect of mitochondrial viability and metabolism on technetium-99msestamibi myocardial retention. Eur JNucl Med 20:20-5.

Dahlberg ST, Leppo JA. (1994) Physiologic properties of myocardial perfusion tracers. Cardiol Clin 12:169-85.

Everett DB, et al. (1977) Gamma-radiation imaging system based on the Compton effect. Proceedings of the IEEE 124:995-1000.

Fallahi B, et al. (2008) Single Tc99m Sestamibi injection, double acquisition gated SPECT after stress and during low-dose dobutamine infusion: a new suggested protocol for evaluation of myocardial perfusion. Int J Cardiovasc Imaging Jun 18. [Epub ahead of print]

Fleming RM, et al. (1990a) Feasibility of SPECT Perfusion Imaging with Technetium 99-m Teboroxime: Comparison to Thallium- 201 and Quantitative Coronary Arteriography. 5th World Congress of Nuclear Medicine and Biology, European eburnal of Nuclear Medicine, Montreal, Canada.

Fleming RM, et al. (1990b) Tc-99m Teboroxime SPECT Imaging: Comparison to Thallium 201 and Quantitative Coronary Arteriography. Circulation 82(4):III-652. 
Fleming RM, et al. (1990c) Nuclear Cardiology and Imaging - Myocardial Scintigraphy II on the Feasibility of SPECT Perfusion Imaging with Technetium 99-m Teboroxime: Comparison to Thallium-201 and Quantitative Coronary Arteriography. XIIth European Heart eburnal 11:277.

Fleming RM, et al. (1991a) A Comparison of Technetium 99-m Teboroxime Tomography to Automated Quantitative Coronary Arteriography and Thallium - 201 SPECT. JAm Coll. Cardiol. 17:1297-1302.

Fleming RM, et al. (1991b) Patterns in visual interpretation of coronary arteriograms as detected by quantitative coronary arteriography. JAm Coll Cardiol 18:945-51.

Fleming RM, Gibbs HR, Swafford J. (1992a) Using Quantitative Coronary Arteriography to Redefine SPECT Sensitivity and Specificity. Am JPhysiol Imag. 7:59-65.

Fleming RM. (1992b) Detecting Coronary Artery Disease Using SPECT Imaging: A Comparison of Thallium-201 and Teboroxime. Am JPhysiol Imag 7(1):20-23.

Fleming RM, Harrington GM. (1994) Quantitative coronary arteriography and its assessment of atherosclerosis. Section II. Calculating stenosis flow reserve from percent diameter stenosis. Angiology 45:835-40.

Fleming RM, Rose CH, Feldmann KM. (1995) Comparing a High Dose Dipyridamole SPECT ImagingProtocol with Dobutamine and Exercise Stress Testing Protocols. Angiology 46(7):547-556.

Fleming RM, Feldmann KM. (1998) Comparing a High Dose Dipyridamole SPECT Imaging Protocol with Dobutamine and Exercise Stress Testing Protocols. Section II: Using High-Dose Dipyridamole to Determine Lung-to-Heart Ratios. Intern JAngiol 7:325328.

Fleming RM. (1999a) Chapter 31. Nuclear Cardiology: Its Role in the Detection and Management of Coronary Artery Disease Textbook of Angiology. John C. Chang Editor, Springer-Verlag New York, NY. pp. 397-406.

Fleming RM, Feldmann KM. (1999b) Comparing a High Dose Dipyridamole SPECT Imaging Protocol with Dobutamine and Exercise Stress Testing Protocols. Section III: Using Dobutamine to Determine Lung-to-Heart Ratios, Left Ventricular Dysfunction and a Potential Viability Marker. Inter JAngiol 8:22-26.

Fleming RM. (1999c) Chapter 64. The Pathogenesis of Vascular Disease. Textbook of Angiology. John C. Chang Editor, Springer-Verlag New York, NY. pp. 787-798.

Fleming RM. (1999d) Chapter 29. Atherosclerosis: Understanding the relationship between coronary artery disease and stenosis flow reserve. Textbook of Angiology. John C. Chang Editor, Springer-Verlag, New York, NY. pp. 381-387.

Fleming RM. (2000a) The Clinical Importance of Risk Factor Modification: Looking at Both Myocardial Viability (MV) and Myocardial Perfusion Imaging (MPI) Intern JAngiol 9:55-69.

Fleming RM. (2000b) The Natural Progression of Atherosclerosis in an Untreated Patient with Hyperlipidemia: Assessment via Cardiac PET. Intern JAngiol 9:70-73.

Fleming RM. (2000c) The Fleming Unified Theory of Vascular Disease: A link between atherosclerosis, inflammation and bacterially aggravated atherosclerosis (BAA). Angiol 51:87-9.

Fleming RM, Boyd L, Forster M. (2000d) Unified Theory Approach Reduces Heart Disease and Recovers Viable Myocardium. 42nd Annual World Congress - International College of Angiology, San Diego, California, USA, June 29, 2000. 
Fleming RM., Boyd L., Forster M. (2000e) Angina is Caused by Regional Blood Flow Differences - Proof of a Physiologic (Not Anatomic) Narrowing, Joint Session of the European Society of Cardiology and the American College of Cardiology, Annual American College of Cardiology Scientific Sessions, Anaheim, California, USA, 12 March 2000, 49th (Placed on internet www.prous.com for physician training and CME credit, April 2000.)

Fleming RM. (2000f) Shortcomings of coronary angiography. Letter to the Editor. Cleve Clin JMed 67:450.

Fleming RM, Boyd L, Forster M. (2000g) Reversing Heart Disease in the New Millennium The Fleming Unified Theory, Angiology 51(10):617-629.

Fleming RM. (2001) Coronary Artery Disease is More than Just Coronary Lumen Disease. Amer JCard 88:599-600.

Fleming RM. (2002a) High-Dose Dipyridamole and Gated Sestamibi SPECT Imaging Provide Diagnositic Resting and Stress Ejection Fractions Useful for Predicting the Extent of Coronary Artery Disease. Angiology 53(4):415-421.

Fleming RM. (2002b) A Tate-en-Tate Comparison of Ejection Fraction and Regional Wall Motion Abnormalities as Measured by Echocardiography and Gated Sestamibi SPECT. Angiology 53:313-321.

Fleming RM. (2002c) Breast enhanced scintigraphy test demonstrates improvement in breast inflammation in women consuming soy protein. The eburnal of Nutrition 132:575S.

Fleming RM. (2002d) Mitochondrial Uptake of Sestamibi Distinguishes Between Normal, Inflammatory Breast Changes, Pre-cancers and Infiltrating Breast Cancer. Integrative Cancer Therapies 1(3):229-237.

Fleming RM, Dooley WC. (2002e) Breast Enhanced Scintigraphy Testing (B.E.S.T.) Distinguishes Between Normal, Inflammatory Breast Changes and Breast Cancer. A Prospective Analysis and Comparison with Mammography. Integrative Cancer Theropies 1(3):238-245.

Fleming RM. (2002f) The Effect of High, Moderate and Low Fat Diets On Weight Loss and Cardiovascular Disease Risk Factors. Preventive Cardiology V(III):110-118. [http://www.medscape.com/viewarticle/438769]

Fleming RM. (2003a) What effect, if any, does soy protein have on breast tissue? Integrative Cancer Therapies 2:225-8.

Fleming RM. (2003b) Are there differences in breast tissue as a result of hormone replacement therapy? Can BEST imaging distinguish these differences? Integrative Cancer Therapies 2:229-34.

Fleming RM. (2003c) Do women taking hormone replacement therapy (HRT) have a higher incidence of breast cancer than women who do not? Integrative Cancer Therapies 2:235-7.

Fleming RM. (2003d) Angina and coronary Ischemia are the result of coronary regional Blood Flow Differences. JAmer Coll Angiol 2003;1:127-42.

Fleming RM. (2003e) Using C-Reactive Protein as a Marker of Bacteriallly Aggravated Atherosclerosis in Acute Coronary Syndromes. JAmer Coll Angiol 1:165-71.

Fleming RM. (2003f) Stop Inflammation Now! with Tom Monte. Published by Putnam Books and Avery Books. December 2003. 
Fleming RM, Harrington GM. (2008) What is the Relationship between Myocardial Perfusion Imaging and Coronary Artery Disease Risk Factors and Markers of Inflammation?, Angiology 59:16-25.

Fleming RM, Harrington GM, Baqir R. (2009) Heart Disease in Men. Chapter 3. Using Multiple Images Post-Stress to Enhance diagnostic Accuracy of Myocardial Perfusion Imaging: The Clinical Importance of Determining Washin and Washout Indicates a Parabolic Function between Coronary Perfusion (Blood Flow) and Cellular ("Uptake/Release") Function. Alice B. Todd and Margo H. Mosley Editors, Nova Publishers pp. 75-100.

(https://www.novapublishers.com/catalog/product_info.php?products_id=8409)

Fleming RM, et al. (2009b) The Evolution of Nuclear Cardiology takes Us Back to the Beginning to Develop Today's "New Standard of Care" for Cardiac Imaging: How Quantifying Regional Radioactive Counts at 5 and 60 Minutes Post-Stress Unmasks Hidden Ischemia. Methodist DeBakey Cardiovascular eburnal (MDCVJ) 5(3):42-48.

Fleming RM, et al.(2010a) Renewed Application of an Old Method Improves Detection of Coronary Ischemia. A Higher Standard of Care. Federal Practitioner 2010;27:22-31.

Fleming RM (2010b) Innovations in myocardial perfusion imaging reveal potential lifesaving strategy. By: Brian Ellis, Cardiology Today, Feature story. 13(9). (http:/ / www.cardiologytoday.com/view.aspx?rid=68069).

Fleming RM, Harrington GM. (2010c) FHRWW Stress SPECT Protocol Reduces Radioactive Dosage and Increases Ischemia Detection. ANZ Nuclear Medicine 41:24-32.

Fukushima K, et al. (2007) Myocardial kinetics of (201) Thallium, (99m) Tc-tetrofosmin, and $(99 \mathrm{~m})$ Tc-sestamibi in an acute ischemia-reperfusion model using isolated rat heart. Ann Nucl Med 21:267-73.

Fujiwara S, et al. (1998) Prediction of functional recovery in acute myocardial infarction: comparison between sestamibi reverse redistribution and sestamibi/BMIPP mismatch. JNucl Cardiol 5:119-27.

Giubbini R, et al. (1995) Evaluation of technetium-99m-sestamibi lung uptake: correlation with left ventricular function. JNucl Med 1995;36:58-63.

Glagov S, et al. (1987) Compensatory enlargement of human atherosclerotic coronary arteries. N Engl JMed 316:1371-5.

Glover DK, et al. (1995) Comparison between 201Tl and 99mTc Sestamibi uptake during adenosine-induced vasodilation as a function of coronary stenosis severity. Circulation 91:813-20.

Goldstein RA, Fleming RM, et al. (1991) SPECT Imaging with Tc99m-teboroxime (TEBO). Japan Society of Nuclear Medicine. elop JNucl Med 28:937.

Gorlin R, et al. (1959) Effect of nitroglycerin on the coronary circulation in patients with coronary artery disease or increased left ventricular work. Circulation 19:705-18.

Gould KL, Kirkeeide RL, Buchi M. (1990) Coronary flow reserve as a physiologic measure of stenosis severity. JAm Coll Cardiol 15:459-74.

Heart (2011) http:/ / www.theheart.org/article/1176107.do

Heisenberg, W. (1927). Ueber den anschaulichen Inhalt der quantentheoretischen Kinematik and Mechanik, Zeitschrift für Physik 43:172-198.

Hurwitz GA, et al. (1993) Pulmonary uptake of sestamibi on early post-stress images: angiographic relationships, incidence and kinetics. Nucl Med Commun 14;15-22. 
Hurwitz GA, et al. (1996) Stress/injection protocols for myocardial scintigraphy with sestamibi and thallium-201: implications of early post-stress kinetics. Nucl Med Commun 17:400-9.

Hurwitz GA, et al. (1998) Pulmonary uptake of Technetium-99m-Sestamibi induced by dipyridamole-basedstress or exercise. JNucl Med 1998;39:339-45.

Husain SS. (2007) Myocardial perfusion imaging protocols: Is there an ideal protocol. $J$ Nucl Med Tech 35:3-9.

Ikawa M, et al. (2007) Evaluation of respiratory chain failure in mitochondrial cardiomyopathy by assessments of 99mTc-MIBI washout and 123I-BMIPP/99mTcMIBI mismatch. Mitochondrion 7:164-70.

Kern MJ, et al. (2006) Physiological assessment of coronary artery disease in the cardiac catheterization laboratory: A scientific statement from the American Heart Association committee on diagnostic and interventional cardiac catheterization, council on clinical cardiology. Circulation 114:1321-41.

Khaw BA. (1999) The current role of infarct avid imaging. Semin Nucl Med 29:259-270.

Kodavanti UP, et al. (2008) The role of Particulate matter-associated zinc in cardiac injury in rats. Environ Health Perspect 116:13-20.

Kumita S, et al. (2002) Assessment of myocardial washout of Tc-99m-sestamibi in patients with chronic heart failure: comparison with normal control. Ann Nucl Med 16:23742.

Lenzi P, et al. (2003) DNA damage associated with ultrastructural alterations in rat myocardium after loud noise exposure. Environ Health Perspect 111:467-71.

Liu Z, et al. (2001) Detection of myocardial viability in ischemic-reperfused rat hearts by Tc99m sestamibi kinetics. JNucl Cardiol 8:677-86.

Love WD. (1965) Isotope Technics in Clinical Cardiology. Circulation 32:309-15.

Maddahi J. (2001) Myocardial perfusion tracers in the detection and evaluation of coronary artery disease. 48 ${ }^{\text {th }}$ annual meeting of the society of nuclear medicine 2001.

Matsunari I, et al. (20010Comparison of Sestamibi, Tetrofosmin, and Q12 Retention in Porcine Myocardium. JNucl Med 42:818-23.

Matsuo S, et al. (2007) A novel clinical indicator using Tc-99m sestamibi for evaluating cardiac mitochondrial function in patients with cardiomyopathies. JNucl Cardiol 14:215-20.

Maublant JC, Gachon P, Moins N. (1988) Hexakis (2-methoxy isobutylisonitrile) technetium-99m and thallium-201 chloride: uptake and release in cultured myocardial cells. JNucl Med 29:48-54.

Meerdink DJ, Leppo JA. (1990) Myocardial transport of hexakis(2-methosyisobutyl isonitrile) and thallium before and after coronary reperfusion. Circulation Research 66:1738-46.

Meissner K, et al. (2002) Expression and localization of P-glycoprotein in Human Heart: Effects of Cardiomyopathy. JHistochem Cytochem 50:1351-6.

Ono S, et al. (2002) Rest delayed images on 99mTc-MIBI myocardial SPECT as a noninvasive screen for the diagnosis of vasospastic angina pectoris. Kaku Igaku 39:117-24.

Ono S, et al. (2003) Enhanced regional washout of technetium-99m-sestamibi in patients with coronary spastic angina. Ann Nucl Med 17:393-8. 
(Oregon 2008) Researchers make case for standardized analysis of cardiac imaging. Oregon Heart \& Vascular Institute, University of Oregon and L.A.'s Cedar-Sinai. March 13, 2008. http://pmr.uoregon.edu

Pace L, et al. (2005) Washout of [99mTc] sestamibi in predicting response to chemotherapy in patients with multiple myeloma. $Q$ JNucl Med Mol Imaging 49:281-5.

Parameswaran RV, Purantharan N, Prasad RD. (2006) Comparison of nitrate augmented 99mTc-Sestamibi infusion with nitrate augmented 99mTc-Sestamibi bolus injection for the detection of myocardial viability. IeNM 21:36-40.

Parkey RW, et al. (1976) Pathophysiology of myocardial infarct scintigraphy with Technetium-99m Pyrophosphate, Tecnnetium-99m Diphosphonate and thallium201. Invest Radiol 11:395.

Poiseuille JLM. (1840) Reherches experimentales sur le mouvement des liquides dans les tubes de tres petits diametres. Comptes Rendus Acad Ac 2:961-1041.

Richter WS, et al. (1995) Washout and redistribution between immediate and two-hour myocardial images using technetium-99m sestamibi. Eur JNucl Med 22:49-55.

Saha M, Forrest TF, Brown KA. (1994) Lung uptake of technetium-99m-sestamibi: relation to clinical, exercise, hemodynamic, and left ventricular function variables. JNucl Cardiol 1:52-6.

Sattari A, Dadashzadeh S, Nasiroghli G. (2001) Urinary excretion and external radiation does from patients administered thallium-201 and technetium-99m methoxy isobutyl isonitrile. Radait Prot Dosimetry 95:59-61.

Sheikine Y, Berman DS, Di Carli MF. (2010) Technetium-99m-sestamibi redistribution after exercise stress test identified by a novel cardiac gamma camera: Two case reports. Clin Cardiol 33:E39-E45.

Shin WJ, et al. (1995) Reverse redistribution on dynamic exercise and dipyridamole stress technetium-99m-MIBI myocardial SPECT. JNucl Med 36:2053-5

Sinusas AJ, et al. (1994) Redistribution of 99m-Tc-sestamibi and 201-Tl in the presence of a severe coronary artery stenosis. Circulation 89:2332-41.

St. Goar FG, et al. (1991) Intravascular ultrasound imaging of angiographically normal coronary arteries: An in vivo comparison with quantitative angiography. IACC 18:952-8.

Sugiura T, et al. (2006) Usefulness of Tc-99m methoxyisobutylisonitrile scintigraphy for evaluating congestive heart failure. JNucl Cardiol 13:64-8.

Takeishi Y, et al. (1996) Reverse redistribution of technetium-99m-sestamibi following direct PTCA in acute myocardial infarction. JNucl Med 37:1289-94.

Takahashi N, et al. (1996) Myocardial uptake of 99m Tc-tetrofosmin, Sestamibi, and $201 \mathrm{Tl}$ in a model of acute coronary reperfusion. Circulation 94:2605-13.

Taki J, et al. (2001) 99mTc-Sestamibi retention characteristics during pharmacologic hyperemia in human myocardium: Comparison with coronary flow reserve measured by Doppler flowire. JNucl Med 42:1457-63.

Tanaka R, et al. (2006) Clinical implication of reverse redistribution on 99mTc-sestamibi images for evaluating ischemic heart disease. Ann Nucl Med 20:349-56.

Toriyama T, et al. (2005) 99mTc-sestamibi scintigraphy in the prediction of cardiac events in patients with congestive heart failure. 7th International conference of Nuclear Cardiology-European Society of Cardiology 2005. 
VanBrocklin HF, et al. (2007) Mitochondrial avid radioprobes. Preparation and evaluation of 7'(Z)-[125I]iodorotenone and 7'(Z)-[125I]iodorotenol. Nucl Med Biol 34:109-16.

Walsh WF, et al. (1977) Assessment of diagnostic value of technetium-99m pyrophosphate myocardial scintigraphy in 80 patients with possible acute myocardial infarction. Br Heart J39:974-981. 


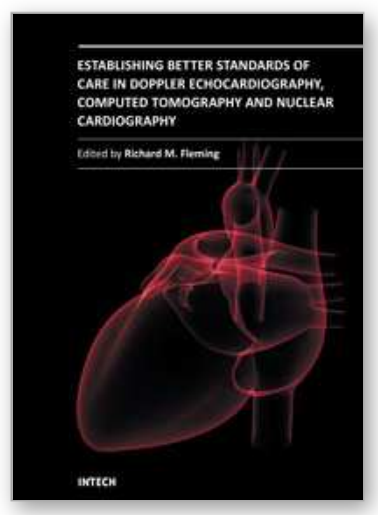

\author{
Establishing Better Standards of Care in Doppler \\ Echocardiography, Computed Tomography and Nuclear \\ Cardiology \\ Edited by Dr. Richard M. Fleming
}

ISBN 978-953-307-366-8

Hard cover, 260 pages

Publisher InTech

Published online 13, July, 2011

Published in print edition July, 2011

Since the introduction of Doppler Echocardiography, Nuclear Cardiology and Coronary CT imaging, clinicians and researchers have been searching for ways to improve their use of these important tools in both the diagnosis and treatment of heart disease. To keep up with cutting edge improvements in these fields, experts from around the world have come together in this book to provide the reader with the most up to date information to explain how, why and when these different non-invasive imaging tools should be used. This book will not only serve its reader well today but well into the future.

\title{
How to reference
}

In order to correctly reference this scholarly work, feel free to copy and paste the following:

Richard M. Fleming and Gordon M. Harrington (2011). Fleming-Harrington Redistribution Wash-in Washout (FHRWW): The Platinum Standard for Nuclear Cardiology, Establishing Better Standards of Care in Doppler Echocardiography, Computed Tomography and Nuclear Cardiology, Dr. Richard M. Fleming (Ed.), ISBN: 978953-307-366-8, InTech, Available from: http://www.intechopen.com/books/establishing-better-standards-ofcare-in-doppler-echocardiography-computed-tomography-and-nuclear-cardiology/fleming-harringtonredistribution-wash-in-washout-fhrww-the-platinum-standard-for-nuclear-cardiology

\section{INTECH}

open science | open minds

\section{InTech Europe}

University Campus STeP Ri

Slavka Krautzeka 83/A

51000 Rijeka, Croatia

Phone: +385 (51) 770447

Fax: +385 (51) 686166

www.intechopen.com

\section{InTech China}

Unit 405, Office Block, Hotel Equatorial Shanghai

No.65, Yan An Road (West), Shanghai, 200040, China

中国上海市延安西路65号上海国际贵都大饭店办公楼 405 单元

Phone: +86-21-62489820

Fax: +86-21-62489821 
(C) 2011 The Author(s). Licensee IntechOpen. This chapter is distributed under the terms of the Creative Commons Attribution-NonCommercialShareAlike-3.0 License, which permits use, distribution and reproduction for non-commercial purposes, provided the original is properly cited and derivative works building on this content are distributed under the same license. 